\title{
Identification of damping and complex modes in structural vibrations
}

\author{
Bajri, Anela; Høgsberg, Jan Becker
}

Published in:

Journal of Sound and Vibration

Link to article, DOI:

10.1016/j.jsv.2018.05.048

Publication date:

2018

Document Version

Peer reviewed version

Link back to DTU Orbit

Citation (APA):

Baji, A., \& Høgsberg, J. B. (2018). Identification of damping and complex modes in structural vibrations. Journal of Sound and Vibration, 431, 367-389. https://doi.org/10.1016/j.jsv.2018.05.048

\section{General rights}

Copyright and moral rights for the publications made accessible in the public portal are retained by the authors and/or other copyright owners and it is a condition of accessing publications that users recognise and abide by the legal requirements associated with these rights.

- Users may download and print one copy of any publication from the public portal for the purpose of private study or research.

- You may not further distribute the material or use it for any profit-making activity or commercial gain

- You may freely distribute the URL identifying the publication in the public portal

If you believe that this document breaches copyright please contact us providing details, and we will remove access to the work immediately and investigate your claim 


\title{
Identification of damping and complex modes in structural vibrations
}

\author{
Anela Bajrića,*, Jan Høgsberg ${ }^{a}$ \\ a Technical University of Denmark, DTU Mechanical Engineering, Nils Koppels Allé Building 403, \\ 2800 Kongens Lyngby, Denmark
}

\begin{abstract}
A sufficiently accurate mathematical representation of the viscous damping matrix from modal parameters is often limited to structures with light damping or an assumed structure of the damping matrix. These limitations are now circumvented by a novel expression, which reconstructs the damping matrix from the complex-valued eigenvectors and eigenvalues of a non-classically damped structure with an assumed mass distribution. The accuracy of this expression is demonstrated by both numerical simulations and experimental measurements of a model-scale five-story shear building, with damping introduced locally by a single eddy current damper. The spatial distribution of the damping is estimated by integrating the proposed expression for the damping matrix in a covariance driven output-only system identification technique. The reproducibility of the mode shape estimates and their convergence with respect to measurement duration validate the proposed approach and demonstrate that complex modes are achievable from vibration measurements.

Keywords: Identification of damping, Complex mode shapes, Random vibrations, Output-only system identification, Local damping sources
\end{abstract}

\section{Introduction}

In complex engineering structures the damping can have spatial sources or variations, which cause transport of vibrational energy through the structure towards the locations where it is dissipated. In the dynamic analysis of structures the damping effect is usually associated with substantial uncertainties. Thus, identification methods have been developed in order to estimate the dynamic properties of structures from vibration measurements [1]. When identifying damping entirely from measurements of the structural response, a classic viscous damping matrix is typically assumed and determined by methods that only use real-valued mode shapes and thereby neglect the above mentioned energy transportation through the structure. A novel method is therefore presented for the estimation of a damping matrix from vibration measurements, which identifies the spatial distribution of the damping sources in the structure.

Experimentally identified mode shapes are commonly complex-valued. Nevertheless, these are often modified in such ways that their real parts are optimally represented [2]. The condition which ensures real-valued mode shapes is defined by Caughey \& O'Kelly's condition of classical damping [3]. Theoretically, the complex-

*Corresponding author: abaj@mek.dtu.dk

Preprint submitted to Elsevier

May 22, 2018 
valued mode shapes appear for linear non-classically damped systems, in which case it turns out that the damping matrix in modal co-ordinates is not diagonal. The off-diagonal terms in the modal damping matrix will couple the modal equations of motion. The complex-valued mode shapes describe an asynchronous motion and are associated with structures where damping can have any spatial distribution [4]. However, there could be other reasons for the inherent complexity of mode shapes identified from measurements, such as measurement errors and signal noise, aliasing and leakage in the signal post-processing, identification errors, system asymmetries from gyroscopic effects or non-linear behavior [2].

The success associated with identifying non-classically damped systems from vibration measurements, is also affected by the specific type of dynamic testing method, the applied identification technique and the subsequent method or expression for constructing the damping matrix. The most common types of dynamic tests are based on impulse, harmonic or random excitation of the structure. They mostly dictate whether an input-output or an output-only identification technique can be applied, and which damping matrix expression can be applied subsequently. The damping identification methods are conveniently categorized as either modal methods based on modal parameters [5-9], local methods that require an equation of motion [10], matrix methods calibrated by frequency response functions [11-13], enhanced methods developed to improve the accuracy of the matrix methods [15] and methods which use transfer function residues [14]. The modal methods are applicable when the response is measured, as well as the case when both the excitation and response are measured. The matrix methods which require the frequency response function or its residues bypass complex mode identification. However they are only applicable when both excitation and response are measured.

The performance of the existing damping identification methods has been demonstrated by numerical simulations with simultaneous knowledge about structural excitation and response and with the presence of noisy data records and modal incompleteness [15-17]. However, the estimation of structural damping has also been investigated experimentally [18], with a given equation of motion and known excitation and response records. For example, complex-valued mode shapes have been experimentally estimated for a plexi-glass plate from free vibrations [19], while it has been shown in [20] that it is difficult to repeatedly identify the complex mode shapes of a free-free beam. The latter has caused a certain skepticism towards the practicality of obtaining complex modes from measurements and the use of modal methods for the identification of structural damping.

The first part of the paper presents a derivation of a novel explitic viscous damping matrix expression. The modal parameters and the mass matrix are required in the construction of the viscous damping matrix by the proposed expression. However, the expression circumvents the requirements of an a priori estimate of the damping matrix in the normalization of the mode shapes and it is in addition not limited to lightly damped systems, as it is the case for the exsisting expressions in $[5,6]$. The efficiency of the proposed damping matrix 
expression combined with an output-only system identification technique is initially explored by numerical simulations. The system identification has been performed by the Eigensystem Realization Algorithm (ERA) [21] on the correlation functions describing the stationary vibrations of a five-story shear building model. The identified damping matrix has been compared with results from Adhikari's modal method [5], which has been found the most effective method in estimating damping [15] and which can furthermore be used in combination with measurements of random structural vibrations.

In the second part of the paper, the identification of damping and complex mode shapes has been obtained from experimental measurements. The test structure is a shear building model identical to that used in the numerical simulations. The experimental data is freely available at [22]. The damping is introduced locally by a single eddy current damper, which can be tuned to range from small to large damping forces and placed at different floors of the building model. Thus, the experimental setup can be altered to provide different shapes of the imaginary part of the estimated mode shapes, which can be related to the location and magnitude of the external damper. The examination of the repeatability of the estimates of complex mode shapes and their convergence with respect to the measurement duration, verify the potential of the proposed damping expression for system identification purposes.

\section{Modal identification methods for viscous non-classical damping}

A multiple-degree-of-freedom system approximates the dynamic response of a continuous structure over a frequency range of interest. The forced response of a viscously damped system with $N$ degrees of freedom can be described by the set of ordinary differential equations

$$
\mathbf{M} \ddot{\mathbf{x}}(t)+\mathbf{C} \dot{\mathbf{x}}(t)+\mathbf{K} \mathbf{x}(t)=\mathbf{F}(t)
$$

where $\mathbf{M}, \mathbf{C}$ and $\mathbf{K}$ are the mass, damping and stiffness matrices, respectively, the associated acceleration, velocity and displacement vectors are represented by $\ddot{\mathbf{x}}, \dot{\mathbf{x}}$ and $\mathbf{x}$, time is $t$ and the external nodal forces are contained in $\mathbf{F}$. While the mass and the stiffness matrices may commonly be derived from kinetic and strain energy functions, the properties of the viscous damping matrix can not be obtained in most situations by first principles and should, therefore, be identified from measurements of dynamic structures.

The free vibrations of the damped structure admit solutions to (1) of the form

$$
\mathbf{x}(t)=\mathbf{\Phi} e^{\Lambda t}
$$

where the complex-valued matrix $\boldsymbol{\Lambda}=\operatorname{diag}\left[\lambda_{1}, \lambda_{2}, \ldots, \lambda_{N}\right]$ contains the eigenvalue $\lambda_{j}$ of vibration mode $j$ in the diagonal. The coefficient matrix $\boldsymbol{\Phi}=\left[\phi_{1}, \phi_{2}, \ldots, \phi_{N}\right]$ contains the complex-valued mode shapes

$\phi_{j}$ as columns. The eigensolution is associated with the eigenvalue problem obtained by the homogeneous form of (1). The free vibration solution (2) is substituted into (1), which determines the following damped 
eigenvalue problem

$$
\left(\mathbf{M} \lambda_{j}^{2}+\mathbf{C} \lambda_{j}+\mathbf{K}\right) \phi_{j}=\mathbf{0} .
$$

The $\lambda_{j}$ and $\phi_{j}$ can be obtained by solving the quadratic eigenvalue problem in (3), as proposed in [23]. However, the objective in the following is to identify the viscous damping matrix $\mathbf{C}$ from the modal properties $\lambda_{j}$ and $\phi_{j}$. This may be an advantage in practical applications, as $\lambda_{j}$ and $\phi_{j}$ can be estimated experimentally by various system identification techniques. Modal methods are therefore considered for the identification of the damping matrix $\mathbf{C}$ in the following.

\subsection{Method in configuration space}

The mode shapes of the damped system can be split into their real and imaginary parts. This is can be written as

$$
\mathbf{\Phi}=\mathbf{U}+i \mathbf{V}
$$

where $i$ is the imaginary unit, while $\mathbf{U}=\left[\mathbf{u}_{1} \mathbf{u}_{2} \ldots \mathbf{u}_{n}\right]$ and $\mathbf{V}=\left[\mathbf{v}_{1} \mathbf{v}_{2} \ldots \mathbf{v}_{n}\right]$ are real-valued $N \times n$ matrices that contain the real and imaginary parts of the complex mode shapes, respectively. The number of modes included in the solution of (1) is often truncated to $n$, which for most practical applications may be substantially smaller than the original dimension $N(n<N)$. By assuming that the elements of $\mathbf{C}$ are small and that the natural frequencies for the modes are well-separated, an approximate expression of the mode shapes can be obtained by a first-order perturbation analysis [24],

$$
\boldsymbol{\phi}_{j}=\mathbf{u}_{j}+i \sum_{k=1}^{N} \frac{\omega_{j} C_{j k}^{\prime}}{\omega_{j}^{2}-\omega_{k}^{2}} \mathbf{u}_{k}\left(1-\delta_{j k}\right),
$$

which is valid for $\omega_{k} \neq \omega_{j}$. Here $C_{j k}^{\prime}=\mathbf{u}_{j}^{T} \mathbf{C} \mathbf{u}_{k}$ is the $\{j, k\}^{\prime}$ th element of the modal damping matrix $\mathbf{C}^{\prime}$, $\omega_{j}=\left|\lambda_{j}\right|$ is the magnitude of the natural frequency and $\delta_{j k}$ is the Kronecker's delta. The approximation in (5) shows that the off-diagonal terms $C_{j k}^{\prime}$ in the modal damping matrix are mathematically responsible for the existence of the imaginary part in the mode shape. The magnitude of the imaginary part in (5) is governed by a factor that is proportional to the product of the natural frequency and the off-diagonal terms in the modal damping matrix, while inversely proportional to the difference between the natural frequencies of modes $j$ and $k$.

From the approximation in (5) an expression to identify the modal viscous damping matrix has been proposed as $[5]$

$$
\mathbf{C}^{\prime}= \begin{cases}\frac{\left(\omega_{j}^{2}-\omega_{k}^{2}\right) D_{j k}}{\omega_{k}} & \text { if } \quad j \neq k \\ 2 \operatorname{Re}\left(\lambda_{j}\right) & \text { if } \quad k=j,\end{cases}
$$

where $\operatorname{Re}(\cdot)$ is the real-valued part of a complex argument, while the matrix $\mathbf{D}$ is given by

$$
\mathbf{D}=\left[\mathbf{U}^{T} \mathbf{U}\right]^{-1}\left[\mathbf{U}^{T} \mathbf{V}\right],
$$


where $(\cdot)^{T}$ denotes the matrix transpose. The expression in (7) can be obtained by assuming that the imaginary parts $\mathbf{V}$ of the complex mode shapes are expanded as a linear combination of the respective real parts $\mathbf{U}$, expressed as $\mathbf{v}_{j}=\sum_{k=1}^{m} D_{j k} \mathbf{u}_{k}$. This assumption and its influence on the damping identification is discussed in $[15,16]$. In (6) the mode shapes $\mathbf{U}$ are normalized according to $\mathbf{u}_{j}^{T} \mathbf{M} \mathbf{u}_{k}=\delta_{j k}$. This implies that the modal mass must be known or experimentally determined. The modal damping matrix in (6) can finally be presented in physical co-ordinates by the transformation

$$
\mathbf{C}=\left[\left(\mathbf{U}^{T} \mathbf{U}\right)^{-1} \mathbf{U}^{T}\right]^{T} \mathbf{C}^{\prime}\left[\left(\mathbf{U}^{T} \mathbf{U}\right)^{-1} \mathbf{U}^{T}\right]
$$

The above damping identification method will in the following be referred to as Adhikari's method. A modified version of this method is provided in [27], which ensures a symmetric viscous damping matrix.

\subsection{Methods in state-space}

The damped eigenvalue problem in (3) is often treated in a state-space format. One of the earliest expressions for the damping coefficient matrix derived from a symmetric state-space format is known as Lancaster's method. The non-symmetric form of the dynamic state matrix is used in the latter as the basis for derivation of a novel explicit expression for the damping matrix that appears to be more robust for larger levels of damping.

\subsubsection{Lancaster's method}

Lancaster's method expresses the damping matrix as

$$
\mathbf{C}=-\mathbf{M}\left(\boldsymbol{\Phi} \boldsymbol{\Lambda}^{2} \boldsymbol{\Phi}^{T}+\overline{\mathbf{\Phi}} \overline{\boldsymbol{\Lambda}}^{2} \boldsymbol{\Phi}^{H}\right) \mathbf{M}
$$

where $(\cdot)^{H}$ is the Hermitian transpose and $(\cdot)$ denotes the complex conjugate. The detailed derivations behind Lancaster's method are provided in $[6,16,25]$. The expression can be used to identify the damping matrix from measured or estimated complex eigenvalues and mode shapes. The mass distribution of the structure should be known and the complex modes must be normalized so that

$$
\phi_{j}^{T}\left(2 \lambda_{j} \mathbf{M}+\mathbf{C}\right) \phi_{j}=1, \quad j=1, \ldots, 2 N
$$

The above set of equations (9) and (10) are unfortunately implicit, as the normalization of the complex modes depends on the damping matrix. An iterative damping matrix identification routine is proposed in [9] based on the method of Lancaster, which involves guessing an initial damping matrix. The computational efficiency of the iterative routine is discussed in [9], where it unfortunately appears that the routine diverges when the damping and mass matrix approach the same order of magnitude. 


\subsubsection{Explicit expression for damping matrix}

This section describes the derivation of the proposed explicit expression for the viscous damping matrix, in which a normalization of the eigenvectors as in (10) is not required, whereby the implicit form of (9) is avoided. Additionally, the assumption of light damping is avoided, which is otherwise the case in (8). The system equations in (1) are initially recast into state-space format,

$$
\dot{\mathbf{z}}(t)=\mathbf{A} \mathbf{z}(t)+\mathbf{B} \mathbf{y}(t),
$$

where $\mathbf{z}$ is the $2 N \times 1$ state vector, $\mathbf{y}$ is a $N \times 1$ input vector, $\mathbf{A}$ is the $2 N \times 2 N$ dynamic state matrix, while $\mathbf{B}$ is the $2 N \times N$ input matrix. The dynamic state matrix of the system in (1) can be written in non-symmetric form,

$$
\mathbf{A}=\left[\begin{array}{cc}
0 & \mathbf{I} \\
-\mathbf{M}^{-1} \mathbf{K} & -\mathbf{M}^{-1} \mathbf{C}
\end{array}\right]
$$

where $\mathbf{0}$ is a null matrix and $\mathbf{I}$ is the corresponding identity matrix. The expression in (12) can be decomposed into block-matrices containing the modal parameters [1],

$$
\mathbf{A}=\Psi \mathbf{L} \Psi^{-1}=\left[\begin{array}{cc}
\Phi & \overline{\boldsymbol{\Phi}} \\
\Phi \Lambda & \overline{\boldsymbol{\Phi}} \bar{\Lambda}
\end{array}\right]\left[\begin{array}{cc}
\Lambda & 0 \\
0 & \bar{\Lambda}
\end{array}\right]\left[\begin{array}{cc}
\Phi & \overline{\boldsymbol{\Phi}} \\
\Phi \Lambda & \overline{\mathbf{\Phi}} \bar{\Lambda}
\end{array}\right]^{-1}
$$

Provided that the block diagonals of $\boldsymbol{\Psi}$ have the dimension $N \times N$, the partitioned inverse of $\boldsymbol{\Psi}$ is

$$
\Psi^{-1}=\left[\begin{array}{cc}
\boldsymbol{\Phi}^{-1}+\boldsymbol{\Phi}^{-1} \overline{\boldsymbol{\Phi}} \mathbf{S} \boldsymbol{\Phi} \Lambda \boldsymbol{\Phi}^{-1} & -\boldsymbol{\Phi}^{-1} \overline{\boldsymbol{\Phi}} \mathbf{S} \\
-\mathbf{S} \boldsymbol{\Phi} \Lambda \boldsymbol{\Phi}^{-1} & \mathbf{S}
\end{array}\right],
$$

where matrices $\mathbf{S}=\left(\overline{\mathbf{\Phi}} \overline{\boldsymbol{\Lambda}}-\boldsymbol{\Phi} \boldsymbol{\Lambda} \boldsymbol{\Phi}^{-1} \overline{\mathbf{\Phi}}\right)^{-1}$ and $\boldsymbol{\Phi}$ are both non-singular. By inserting (14) into (13) the dynamic state matrix is re-formulated as

$$
\mathbf{A}=\left[\begin{array}{cc}
\mathbf{0} & \mathbf{I} \\
\left(\boldsymbol{\Phi} \Lambda^{2} \boldsymbol{\Phi}^{-1}\left(\mathbf{I}+\overline{\mathbf{\Phi}} \mathbf{S} \boldsymbol{\Phi} \Lambda \boldsymbol{\Phi}^{-1}\right)-\overline{\mathbf{\Phi}} \bar{\Lambda}^{2} \mathbf{S} \boldsymbol{\Phi} \Lambda \boldsymbol{\Phi}^{-1}\right) & \left(\overline{\mathbf{\Phi}} \bar{\Lambda}^{2}-\mathbf{\Phi} \Lambda^{2} \boldsymbol{\Phi}^{-1} \overline{\boldsymbol{\Phi}}\right) \mathbf{S}
\end{array}\right],
$$

which is an expression for the dynamic matrix in terms of the modal parameters. Finally, when equating (12) and (15) the damping matrix can be expressed as

$$
\mathbf{C}=\mathbf{M}\left(\boldsymbol{\Phi} \Lambda^{2} \boldsymbol{\Phi}^{-1} \overline{\boldsymbol{\Phi}}-\overline{\mathbf{\Phi}} \bar{\Lambda}^{2}\right) \mathbf{S}
$$

This expression implies that the damping matrix can be obtained by the mode shapes, eigenvalues and the mass matrix of the system, where the mode shapes in this expression are not dependent on a particular scaling. By dynamic testing of structures that are of a reasonable size and accessibility, and by subsequently performing experimental modal analysis it is possible to obtain the mode shapes and eigenvalues as well as the mass matrix [26]. For structures which are e.g. large in scale or complex in geometry, the mass matrix 
Table 1: Computational procedure for identification of damping from structural vibrations to random excitation.

1. Compute and arrange the auto- and cross-correlation function estimate in a matrix $\mathbf{R}(\tau)$ from measured random structural vibrations $\mathbf{x}(t)$ at a number of spatial locations.

2. Use correlation driven output-only system identification procedure to estimate the complex poles $\boldsymbol{\Lambda}$ and complex mode shapes $\boldsymbol{\Phi}$ for a fixed model order.

3. Compute the matrix: $\mathbf{S}=\left(\overline{\mathbf{\Phi}} \bar{\Lambda}-\boldsymbol{\Phi} \Lambda \boldsymbol{\Phi}^{-1} \overline{\boldsymbol{\Phi}}\right)^{-1}$.

4. Represent the structure by a discrete numerical model and determine a mass matrix: $\mathbf{M}$.

5. Determine the damping matrix: $\mathbf{C}=\mathbf{M}\left(\mathbf{\Phi} \Lambda^{2} \boldsymbol{\Phi}^{-1} \overline{\boldsymbol{\Phi}}-\overline{\mathbf{\Phi}} \bar{\Lambda}^{2}\right) \mathbf{S}$.

may instead be reliably computed by a numerical Finite Element model. An expression for the stiffness matrix in terms of modal parameters can similarly be found as

$$
\mathbf{K}=\mathbf{M}\left(\overline{\boldsymbol{\Phi}} \bar{\Lambda}^{2} \mathbf{S} \boldsymbol{\Phi} \Lambda \boldsymbol{\Phi}^{-1}-\boldsymbol{\Phi} \Lambda^{2} \boldsymbol{\Phi}^{-1}\left(\mathbf{I}+\overline{\boldsymbol{\Phi}} \mathbf{S} \boldsymbol{\Phi} \boldsymbol{\Lambda} \boldsymbol{\Phi}^{-1}\right)\right)
$$

although the present paper mainly focuses on damping identification. The expression for the stiffness matrix may be informative in other applications. For an incomplete set of eigenvalues and eigenvectors obtained from a limited frequency range the expression in (16) yields a damping matrix of reduced accuracy. Thus, a full set of eigenvalues and eigenvectors are required to reproduce the exact damping matrix. Modal incompleteness is discussed in relation to modal damping identification methods in [15-17], while the influence of modal incompleteness on the specific expression in (16) has not yet been investigated. The derived expression (16) is now integrated in an output-only system identification technique, as summarized in Table 1. It should be noted that the derived expression in (16) is not limited to output-only techniques.

A conservative system has been assumed in the derivation of the expression for the damping matrix. However, erroneously identified modal parameters from experimental data in combination with the expression in (16) does not guarantee a symmetric damping matrix, in such case the methodology provided in [27] is advantageous as it will guarantee symmetry of the identified damping matrix, however it is limited to lightly damped systems.

The input to the identification procedure in Table 1 is the estimated correlation function matrix of the structural response to white noise excitation. The most common correlation driven techniques, which can be incorporated in step 2 of Table 1, include the ERA, the Ibrahim Time Domain identification and the Covariance driven Stochastic Subspace Identification, see details in [21, 30, 31]. In the present analysis the ERA technique described in [21] has been implemented to provide the complex-valued modal parameters required in step 2 of the damping identification method in Table 1. Recall that the angular natural frequencies and modal damping ratios of mode $j$ are obtained from the complex roots $\lambda_{j}$ by the relations $\omega_{j}=\left|\lambda_{j}\right|$ and $\zeta_{j}=-\frac{\operatorname{Re}\left(\lambda_{j}\right)}{\left|\lambda_{j}\right|}$, respectively. The magnitude of the identified damping by the final step of the procedure in 
(a)

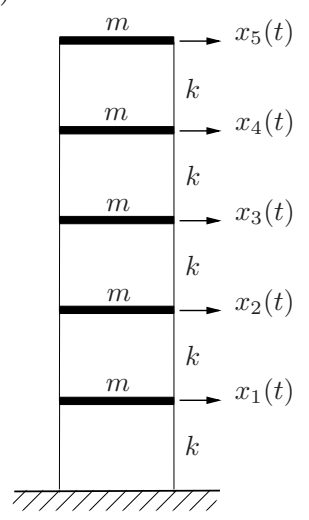

(b)

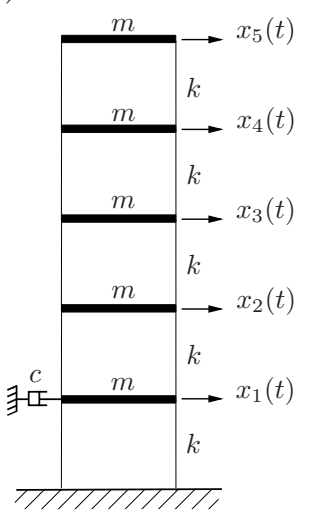

(c)

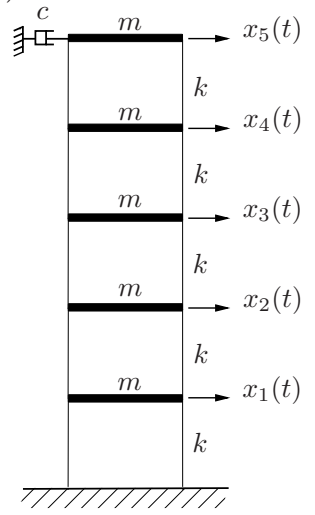

Figure 1: (a) A five-story shear building model with (b) a viscous damper attached at the first floor and (c) a viscous damper attached at the fifth floor.

Table 1 may deviate depending on the choice of system identification technique, as investigated for real-valued mode shapes in [32-34].

\section{Numerical analysis of shear building model}

This initial numerical example demonstrates the proposed damping expression incoorporated in an identification procedure applied on the five-story shear building model shown in Figure 1(a). The governing equation of motion of the structure is expressed as in (1). For this shear building model, the mass matrix $\mathbf{M}$ and stiffness matrix $\mathbf{K}$ are simply constructed as

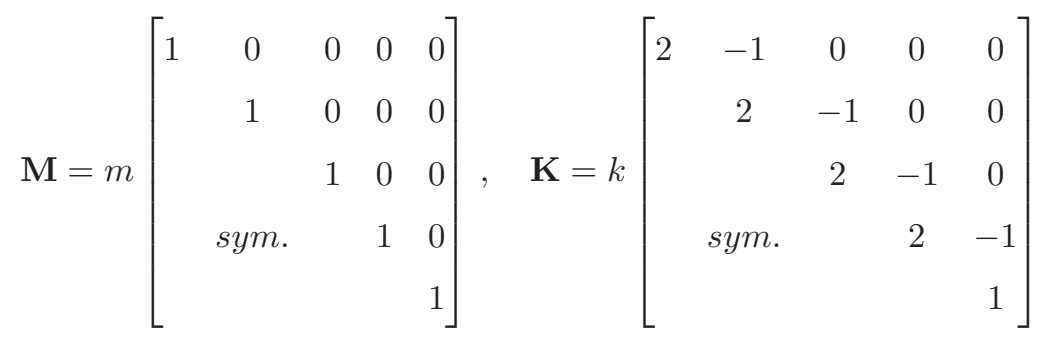

with floor mass $m=2.2 \mathrm{~kg}$ and inter-story stiffness $k=3000 \mathrm{~N} / \mathrm{m}$ chosen so that the properties of this linear model are comparable to those of the physical model used in the experimental analysis in Section 5.

Supplemental damping is introduced by a single local damper which acts on the absolute motion of a particular building floor, as indicated in Figure 1(b)-(c). Thus, the total damping of the structural model is composed of the inherent structural damping and the additional contribution from this external damper. The damping matrix can therefore be expressed as [35]

$$
\mathbf{C}=\tilde{\mathbf{C}}+c \mathbf{w} \mathbf{w}^{T},
$$


where $\tilde{\mathbf{C}}$ is the structural damping matrix, $c$ is the viscous coefficient of the external damper and $\mathbf{w}$ is the connectivity vector that determines the location of the damper on the building. The structural damping matrix $\tilde{\mathbf{C}}$ is in the numerical analysis constructed as

$$
\tilde{\mathbf{C}}=\left[\begin{array}{ccccc}
\tilde{c}_{1} & -\tilde{c}_{2} & 0 & 0 & 0 \\
& \tilde{c}_{1} & -\tilde{c}_{2} & 0 & 0 \\
& & \tilde{c}_{1} & -\tilde{c}_{2} & 0 \\
& \text { sym. } & & \tilde{c}_{1} & -\tilde{c}_{2} \\
& & & & \tilde{c}_{3}
\end{array}\right] .
$$

where the components of the damping matrix obtained by $\tilde{c}_{1}=0.3 \mathrm{Ns} / \mathrm{m}, \tilde{c}_{2}=0.12 \mathrm{Ns} / \mathrm{m}$ and $\tilde{c}_{3}=0.2 \mathrm{Ns} / \mathrm{m}$ agree well with the experimentally identified values in Section 5. In which case $\tilde{\mathbf{C}}$ provides proportional damping. The identification procedure of Table 1 has been performed for the three cases shown in Figure 1: (a) without an external damper, (b) a viscous damper on the first floor and (c) a viscous damper on the fifth floor. For case (b) the connectivity vector is $\mathbf{w}=[1,0,0,0,0]^{T}$, while for (c) it is changed to $\mathbf{w}=[0,0,0,0,1]^{T}$. The viscous coefficient $c$ of the external damper is in the following modified depending on the placement of the damper.

The identification procedure in Table 1 starts by the correlation function. In this example the correlation function matrix of the building model has been simulated by the expression [29]

$$
\mathbf{R}(\tau)=2 \pi \sum_{n=1}^{N}\left(\mathbf{A}_{n} \mathbf{G} \boldsymbol{\Phi}_{n} e^{-\lambda_{n} \tau}+\overline{\mathbf{A}}_{n} \mathbf{G} \overline{\mathbf{\Phi}}_{n} e^{-\bar{\lambda}_{n} \tau}\right),
$$

where the exterior product of a mode shape vector

$$
\mathbf{A}_{n}=\frac{\phi_{n} \phi_{n}^{H}}{m_{n}}
$$

is normalized by the modal mass $m_{n}$ of mode $n$, while $\mathbf{G}$ is a positive definite covariance matrix. The expression in (21) shows that the correlation function matrix is the sum of the same type of decaying harmonics as the impulse response function of single harmonic oscillators. This similarity enables the application of correlation driven time domain output-only system identification techniques. The time delay $\tau=n \Delta \tau$ has been determined by the increment $\Delta \tau=100$ s and step $n=0,1,2, \ldots, 600$, whereby the number of lags included in the identification procedure capture the first ten periods of the first vibration mode. The constant multiplier $\mathbf{G}$ has been generated by obtaining the eigenvalues $\boldsymbol{\Lambda}_{G}$ and eigenvectors $\boldsymbol{\Phi}_{G}$ of a $5 \times 5$ matrix consisting of pseudo-random numbers with a Gaussian distribution. The eigenvalues have been restricted to positive values only, hence $\mathbf{G}=\boldsymbol{\Phi}_{G} \boldsymbol{\Lambda}_{G} \boldsymbol{\Phi}_{G}^{-1}$ is positive definite. The system identification technique has been chosen as the ERA method, implemented in MATLAB following the original formulation in [21]. The model order was chosen as 10, which is twice the number of degrees of freedom in the present structural model. The expression in (16) may be sensitive to estimation errors in the modal parameters, due to the 
computation of the inverse. Therefore, the damping matrix identified by the proposed direct method is in the following numerical examples compared to the damping matrix identified by Adhikari's method, based on the expression in (8). The procedure for Adhikari's method is summarized in [5] and is herein combined with the modal parameters obtained by step 1 and 2 in Table 1.

\subsection{Results for low damping}

Initially, relatively small levels of external damping have been introduced by $c=2.8 \mathrm{Ns} / \mathrm{m}$ for the damper acting on the first floor in case (b) and $c=4.8 \mathrm{Ns} / \mathrm{m}$ for case (c) with the damper acting on the top floor, see Figure 1. The total damping matrix defined in (19) is now identified using both Adhikari's methods based on the expression in (8) and the proposed explicit expression represented by (16). The identified damping matrices $\hat{\mathbf{C}}$ are denoted by $(\hat{\cdot})$ and shown in Figure 2 for the three damping cases given in Figure 1(a)-(c). The individual components $\hat{C}_{j k}$ of the identified damping matrix $\hat{\mathbf{C}}$ from the present method are shown in Figure 2(a)-(c). It is initially found that the identified damping matrix is symmetric for both the proposed expression in (16) and the method of Adhikari, which is expected from numerical simulations as the identified complex modes and complex roots are error free.

The deviation between the actual damping matrix $\mathbf{C}$ and the identified matrix $\hat{\mathbf{C}}$ is evaluated by the relative error
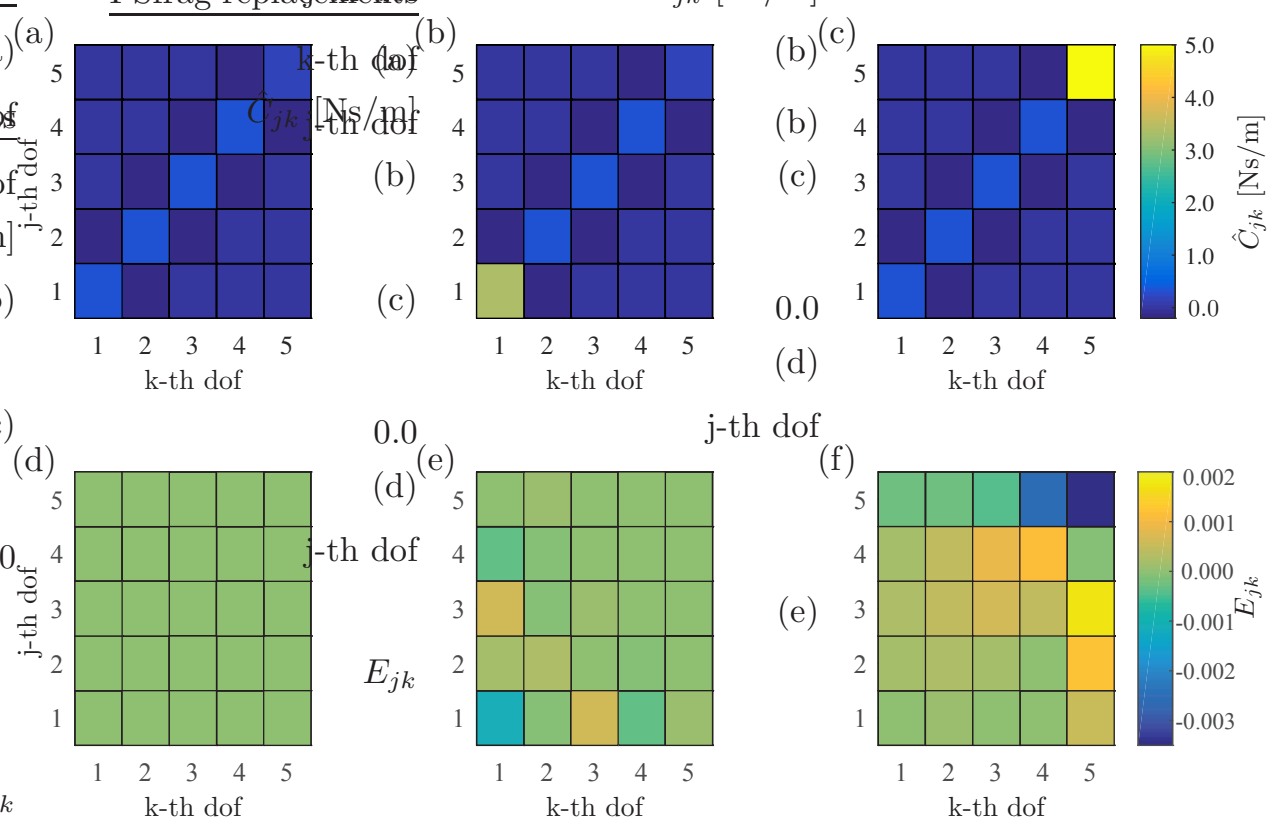

Figure 2: Components $\hat{C}_{j k}$ of the estimated damping matrix $\hat{\mathbf{C}}$ by the proposed explicit expression in (16) including (a) structural damping, (b) damper on first floor and (c) damper on fifth floor. Components $E_{j k}$ of the relative error matrix $\mathbf{E}$ for the identified damping by Adhikari's method for (d) structural damping, (e) damper on first floor and (f) damper on fifth floor. 


$$
\mathbf{E}=\frac{\hat{\mathbf{C}}-\mathbf{C}}{\|\mathbf{C}\|}
$$

where $\|(\cdot)\|$ is the matrix 2-norm. It is seen from the results in Figure $2(\mathrm{~b})-(\mathrm{c})$ that the present identification method clearly indicates the local damper placed on the first floor in (b) and top floor in (c), and the colorcodes indicate that $\hat{C}_{55}=3 \mathrm{Ns} / \mathrm{m}$ and $5 \mathrm{Ns} / \mathrm{m}$ are reproduced with good agreement.

The estimated values from the present direct method are in fact so accurate that the error in $\mathbf{E}$ is less than $10^{-8}$ for all three cases (a)-(c). In Figure 2 (d) -(f) the error in (23) is shown for $\hat{\mathbf{C}}$ obtained by Adhikari's method. It appears that this method also exactly estimates case (a) with proportional damping and without an external damper. However, the relative error matrix $\mathbf{E}$ in Figure 2 (e)-(f) for cases (b) - (c) with a local damper shows that Adhikari's method underestimates the magnitude of the viscous damper, represented by the negative error measures at the location of the external damper. The damping is however overestimated in the proximity of the viscous damper, as indicated by the positive errors in (e) and (f).

\subsection{Results for larger damping}

The performance of the damping identification procedure in Table 1 is now evaluated for larger values of the viscous coefficient $c$ for the external damper. This analysis only considers case (c) in Figure 1 with a damper acting on the absolute motion of the top floor. The simulation parameters and identification technique specifications are however unchanged. The viscous coefficient of the damper at the top floor is now varied from $c=0$ to $49.8 \mathrm{Ns} / \mathrm{m}$, such that the component $C_{55}$ is within the range from 0 to $50 \mathrm{Ns} / \mathrm{m}$. Based on a full complex eigenvalue analysis of the damped system, it has been found that maximum attainable modal damping ratio of the first mode is obtained when tuning the viscous coefficient of the damper to approximately $c=100 \mathrm{Ns} / \mathrm{m}$, which means that the considered interval of $c$ is a suitable compromise between large levels of attainable damping and sufficient motion of the top floor in the simulations. The modal damping ratio of the first mode varies from the level of pure structural damping with $\zeta_{1}=0.016$ obtained for $c=0 \mathrm{Ns} / \mathrm{m}$ to the largest damping ratio $\zeta_{1}=0.3951$ within the simulation study obtained at $c=49.8 \mathrm{Ns} / \mathrm{m}$. Thus, very large levels of attainable modal damping have been achieved.

The identified damping is assessed in terms of the fifth diagonal element of the identified damping matrix $\hat{C}_{55}$, associated with the location of the external damper. The variation of the estimated damping matrix component $\hat{C}_{55}$ is shown in Figure 3 (a), as a function of the component $C_{55}$. The blue curve is obtained by the present direct identification method, while the black curve represents the method of Adhikari. Both methods generally provide good results. However, while the proposed method maintains a straight (blue) line over the full interval of $C_{55}$, the benchmark method causes a notable underestimation for larger values of $C_{55}$ and for $C_{55}=50 \mathrm{Ns} / \mathrm{m}$ it provides a relative error of $10 \%$.

Another assessment of the identification accuracy is in Figure 3(b) demonstrated by the norm of the error matrix $\|\mathbf{E}\|$. It indicates that the error of the proposed normalization free direct method (blue curve) is very 
small and without any increase for larger damper magnitudes, whereas it is initially larger and increases monotonically for the benchmark method.

\section{Dynamic testing of a five-story shear building model}

The dynamic testing of a five-story shear building model has been performed to demonstrate the ability of the damping identification method to determine the complex modes and the damping matrix from actual measurements of the floor displacements. A schematic illustration of the experimental setup and the associated hardware/software connections is provided in Figure 4, while Figure 5 shows a photo of the setup in the laboratory.

The shear building model structure is placed on a plate with a mass of $30 \mathrm{~kg}$ which slides on two rails fixed to a strong table. The horizontal position of the plate is controlled by an electromagnetic motor consisting of a stator and slider, connected to a servo driver and power supply. The plate, rails and motor together comprise a shaking table, used to excite the shear building model. The desired motion profile of the shaking table is fed to the servo drive of the motor as an analog signal generated by a normally distributed pseudo-random sequence regarded as white noise within the frequency range of the structure. The excitation signal $f(t)$ has been generated in LabView from the expression [37]

$$
f(t)=\sum_{k=-p / 2+1}^{p / 2-1} F_{k} e^{i\left(2 \pi k \frac{f_{s}}{p} t+\theta_{k}\right)},
$$

where $F_{k}=F_{-k}$ adjusts the amplitude with $F_{0}=0, p$ is the number of points, $f_{s}$ is the sampling frequency and $\theta_{k}=\theta_{-k}$ is the temporal phase randomly generated by a uniform distribution between 0 and $2 \pi$. Thus, the excitation signal $f(t)$ becomes asymptotically Gaussian-distributed for an increasing number of samples $p$.

(a)

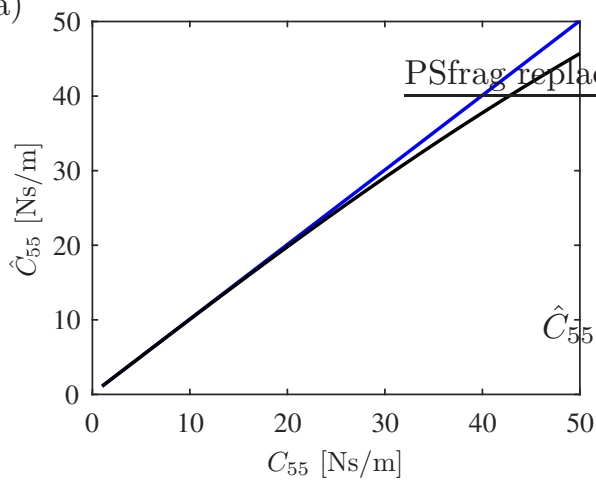

(b)

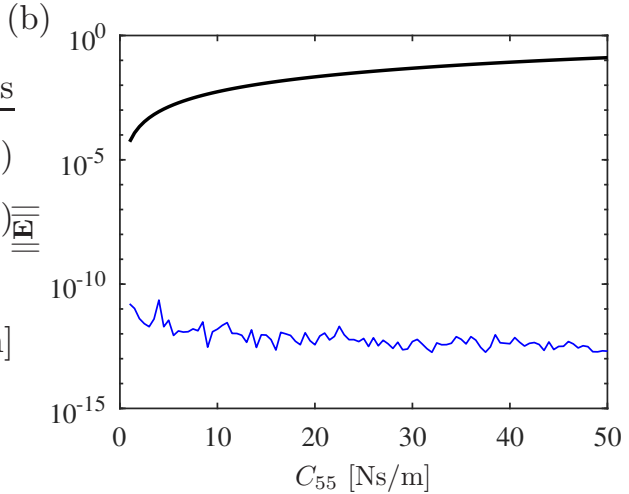

Figure 3: Identified damping by Adhikari's method (-) and the proposed expression (-) for damper on fifth floor with varying magnitude. (a) Estimated component $\hat{C}_{55}$ of damping matrix associated with fifth floor and (b) the norm $\|\mathbf{E}\|$ of the relative error matrix. 
The building model consists of five rectangular aluminum plates $(200 \mathrm{~mm} \times 240 \mathrm{~mm} \times 15 \mathrm{~mm})$ connected by four stainless steel columns $(0.8 \mathrm{~mm} \times 30 \mathrm{~mm} \times 1000 \mathrm{~mm})$, see Figures $4-7$. Rigid connections have been ensured between the floors and columns by additional steel clamps of the same thickness as the floor plates and a the sixth bottom plate secures a rigid connection to the shaking table. The mass of a single floor including connections and bolts is $2.13 \mathrm{~kg}$. The absolute displacement of each floor is measured by five individual laser optical displacement sensors, with a measurement range of $50 \mathrm{~mm}$. In Figure 4 these sensors are denoted 1 (top) to 5 (bottom), which are mounted on a common steel rack fixated to the strong table. Sensor 6 is mounted directly on the strong table. It has a range of $20 \mathrm{~mm}$ and is used to measure the displacement of the shaking table plate. The sensors are connected to the same data acquisition module by BNC cables. The data acquisition module simultaneously converts the analog signal from the sensors to a digital signal for each of the six channels. Thus, the displacement measurements are recorded with a sampling frequency of $100 \mathrm{~Hz}$ without any time delay between measurement channels. The duration of each measurement campaign is 12 hours, corresponding to [38]

$$
T_{n}=\frac{200}{f_{n} \zeta_{n}}
$$

which is the measurement time necessary when assuming that the fundamental natural frequency and damp-

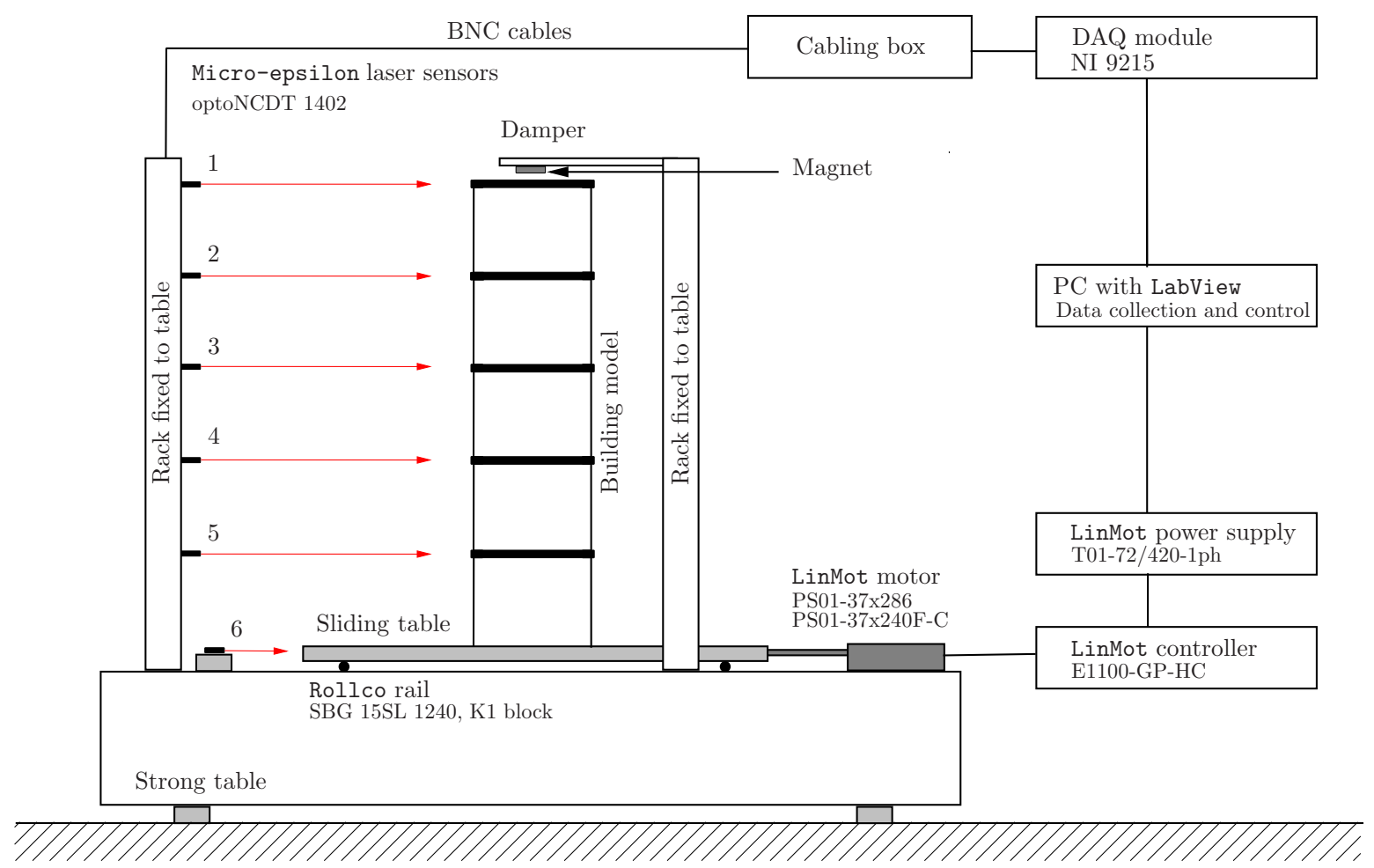

Figure 4: Sketch of the experimental setup of the building model. 


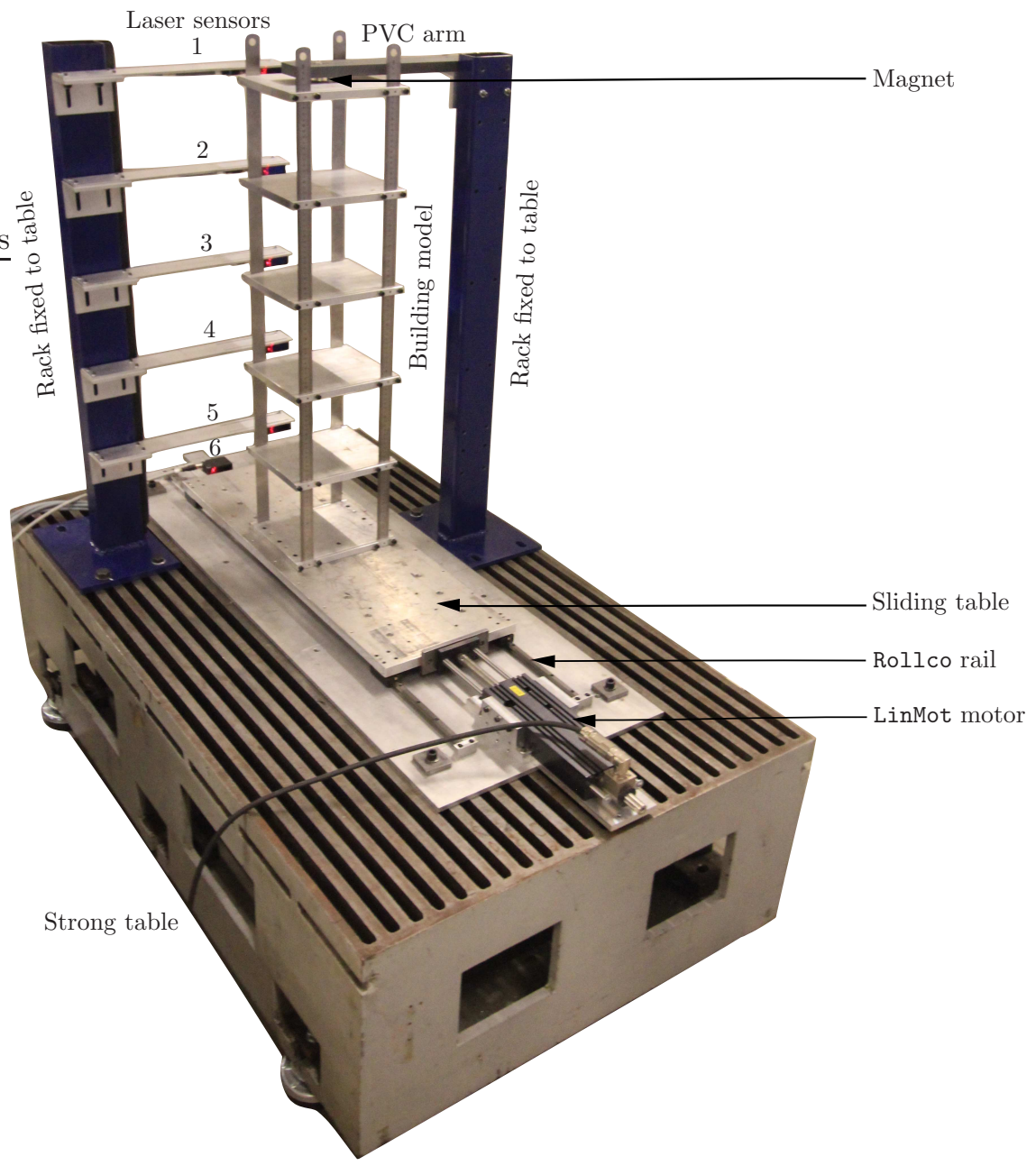

Figure 5: Photo of the experimental setup of the building model.

ing of the building models are approximately $f_{1}=1.6 \mathrm{~Hz}$ and $\zeta_{1}=0.003$.

The local damping source was induced by an eddy current damper. The damper consists of a cylindrical Neodymium magnet, fixed to an arm of PVC material. This ensures that no significant effects from the mounting disturb the magnetic field. The arm is fixed to a separate steel rack. The setup allows the floor to act as a conductor which experiences a time varying magnetic flux due to the relative movement of the conductor orthogonal to the poling direction of the magnetic field. The eddy currents in the conductor generate an electromotive force proportional to the velocity of the particular building floor and the force can, therefore, be characterized as a drag force acting as a desired viscous damper element. The resistance of the conductor will cause the energy to be dissipated as heat. Figure 6 shows the position of the magnet and conductor, and Figure 7 shows a photo of the magnet acting on the top floor of the building model. The magnetization strength of the magnet has been $J=1.22 \mathrm{~T}$ which is specified by the manufacturer. The thickness $t_{m}=8 \mathrm{~mm}$, inner radius $a_{1}=2.75 \mathrm{~mm}$ and outer radius $a_{2}=20 \mathrm{~mm}$ specify the geometry of the 
(a)

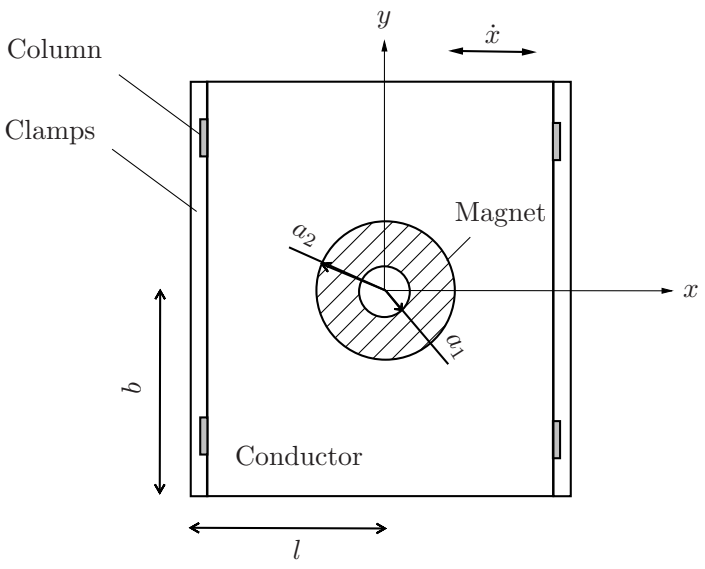

(b)

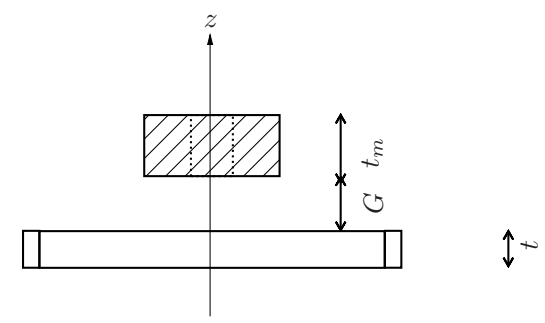

Figure 6: Geometry of the eddy current damper consisting of a cylindrical magnet and a rectagular plate conductor. (a) Plan view, and (b) side view of the damper setup.

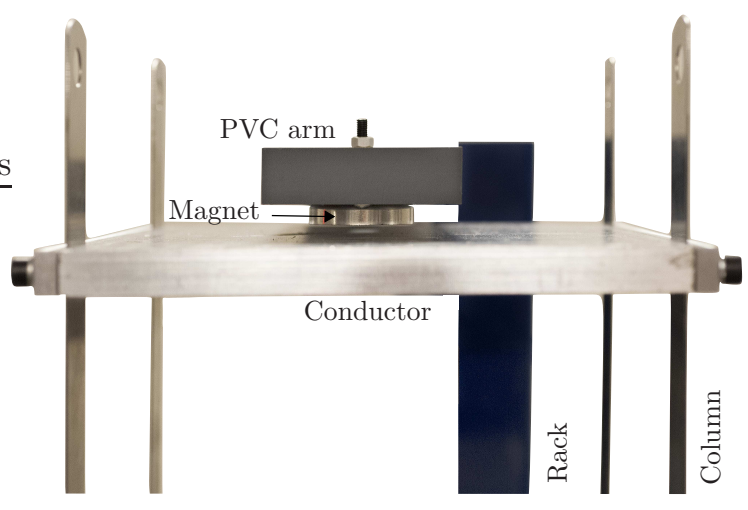

Figure 7: Photo of the eddy current damper setup placed at the fifth floor.

magnet. The conductor has an electrical resistivity of $\rho=2.835 \times 10^{-8} \Omega \mathrm{m}$, thickness of $t=15 \mathrm{~mm}$, length $l=108 \mathrm{~mm}$ and width $b=120 \mathrm{~mm}$, as shown in Figure 6 . The damper force of a single magnet with an air gap $G=2 \mathrm{~mm}$ to $6 \mathrm{~mm}$ with the specified conductor is expected in the range $1 \mathrm{Ns} / \mathrm{m}$ to $2 \mathrm{Ns} / \mathrm{m}$ [36]. The influence of the steel columns on the damping force is neglected, and the clamps are regarded as conductors with a thickness equivalent to the floor plate.

\section{Experimental results of the spatial damping identification}

The test data consists of random vibration measurements from the six displacement sensors placed next to the building model, as illustrated in Figure 4. The experimental data which reproduce the results presented in the following are available at [22]. In the following the eddy current damper is either placed at the first (bottom) floor or the fifth (top) floor. The identification procedure summarized in Table 1 is used to identify 
the modal parameters and the damping matrix from the measurements obtained by the conducted dynamic tests. The parameters of the identification procedure are identical to those applied in the numerical study in Section 3.

The basis of the damping matrix identification is the preliminary estimation of the modal parameters, which are determined from the correlation functions of the measured displacement records using an unbiased correlation function estimator, as described in [1]. Several correlation function estimators exist for vibration measurements of finite duration. A more detailed assessment and comparison of these different estimators have been conducted in [28].

Figure 8 shows the response from measurements obtained for the case (a) without the external damper. Figure 8 (a) shows the measured displacement, while Figure 8 (b) shows the auto-correlation functions estimated from the measured displacements. The auto-correlation functions for the individual floor displacements (sensors 1 to 5) correspond to exponentially decaying oscillations, while the correlation function for the shaking table displacement (sensor 6) exhibits a spike at zero time lag and subsequent small oscillations. The latter verifies the intended white noise excitation caused by the position control of the shaking table actuator. It should be noted that the measured displacement of the shaking table has not been used in the present output-only identification procedure.

Figure 8 (c) shows the auto-power spectral densities obtained for the six displacement signals. The resonance peaks of the building model are clearly visible in the frequency range up to $12 \mathrm{~Hz}$. However, it is found that the resonance peak of mode four is not captured very well by sensor 3 due to an anti-resonance point at this location in mode four. The consequence of this with respect to the estimation of the damping matrix is discussed in Section 6. The spectrum of the shaking table displacement (sensor 6) has a maximum at around a corner frequency of $14 \mathrm{~Hz}$, above which the energy content in the signal decreases gradually. Below the corner frequency the spectrum of the shaking table displacement is fairly flat and the actuator is therefore able to reproduce the desired shaking table motion within the relevant frequency range of the building model. However, small peaks are visible at each resonance frequency, which occur due to the inevitable dynamic coupling between the building model and the shaking table.

Table 2 summarizes the natural frequencies and the damping ratios for the first five vibration modes of the building model, obtained by the present identification procedure in Table 1 on the basis of the estimated correlation functions from the measured vibrations. The first column in the table presents the identified natural frequencies for the building model without damper. The second column of the table gives the coefficient $\gamma_{n}=\frac{f_{n}}{f_{n}-f_{n-1}}$ of the modal overlap factor, $\mu_{n}=\zeta_{n} \gamma_{n}$, which describes the ratio of the modal bandwidth relative to the separation of the natural frequencies. Low values of $\mu_{n}$ indicates that the modes are well separated, in which case the identification errors are expected to be small, while an increase in modal overlap concurrently results in mode shapes with a significant imaginary part [39]. The building 

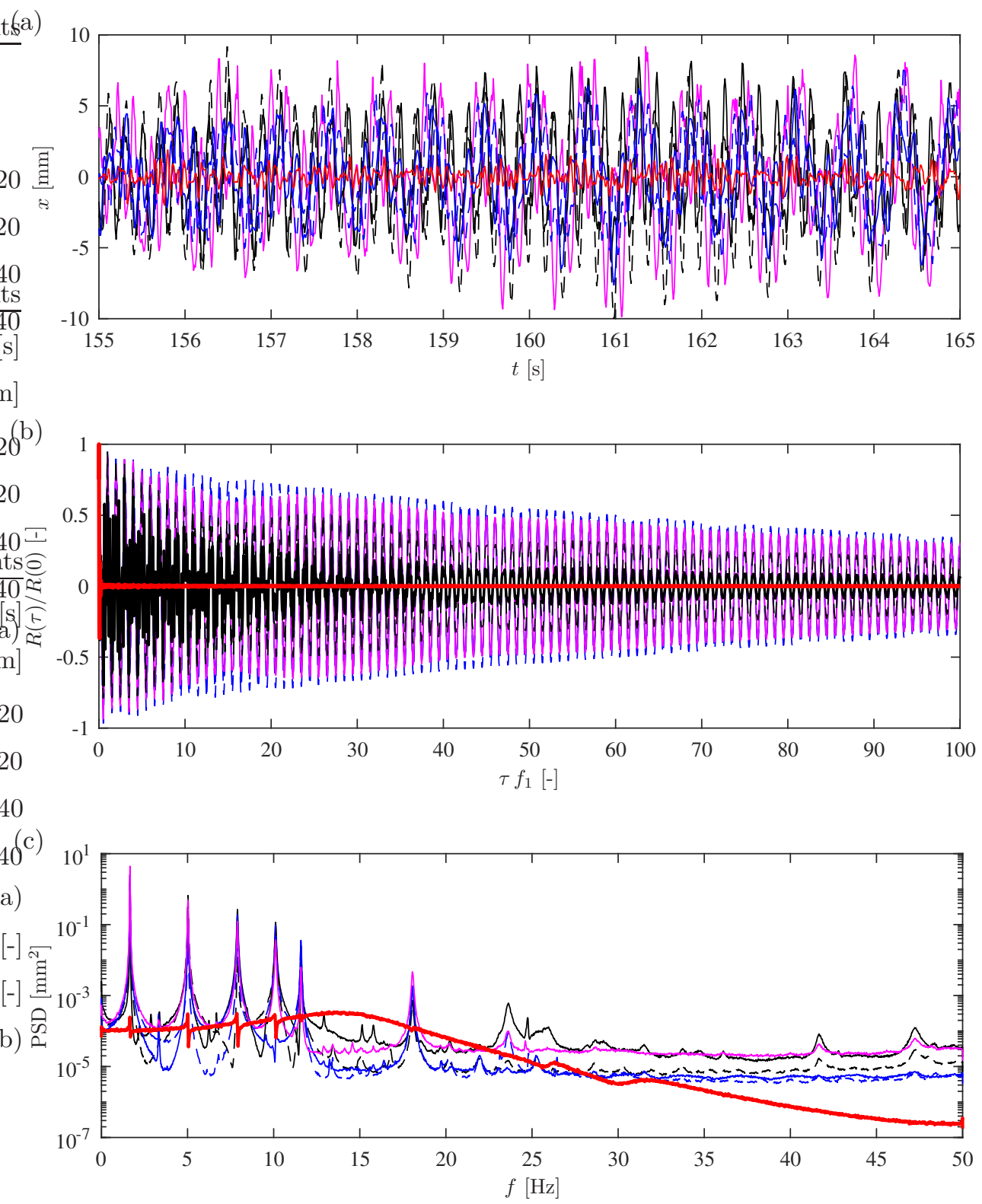

Figure 8: (a) Measured displacement, (b) auto-correlation function estimate and (b) auto-power spectral density (PSD) of the measured signal from sensor $1(-)$, sensor $2(--)$, sensor $3(-)$, sensor $4(--)$, sensor $5(-)$, sensor $6(-)$.

model in this study has a relatively low modal overlap without the damper, and increasing values of modal overlap when the external damper is attached. Thus, an increase in the imaginary part of the mode shapes is expected with an increasing damper force.

The last column of the table shows the associated damping ratios for the building structure without damper.

The building model is lightly damped with modal damping ratios below $0.3 \%$ of critical. The table also presents the identified modal damping ratios obtained for an experimental setup with the eddy current 
Table 2: Estimated natural frequencies, damping ratios and coefficient of the modal overlap factor with no damper, damper at the first floor and damper at the fifth floor with an air gap size of $2 \mathrm{~mm}$ to $6 \mathrm{~mm}$.

\begin{tabular}{cccccccccc}
\hline$n$ & $f_{n}[\mathrm{~Hz}]$ & $\gamma_{n}$ & \multicolumn{3}{c}{ First floor, $\zeta_{n}$} & \multicolumn{3}{c}{ Fifth floor, $\zeta_{n}$} & $\zeta_{n}$ \\
& - & - & $2 \mathrm{~mm}$ & $4 \mathrm{~mm}$ & $6 \mathrm{~mm}$ & $2 \mathrm{~mm}$ & $4 \mathrm{~mm}$ & $6 \mathrm{~mm}$ & - \\
\hline 1 & 1.66 & - & 0.0028 & 0.0022 & 0.0019 & 0.0168 & 0.0122 & 0.0092 & 0.0015 \\
2 & 5.02 & 1.4940 & 0.0055 & 0.0042 & 0.0034 & 0.0060 & 0.0050 & 0.0043 & 0.0023 \\
3 & 7.90 & 2.7431 & 0.0067 & 0.0050 & 0.0040 & 0.0044 & 0.0039 & 0.0035 & 0.0028 \\
4 & 10.11 & 4.5747 & 0.0053 & 0.0040 & 0.0034 & 0.0034 & 0.0031 & 0.0029 & 0.0026 \\
5 & 11.59 & 7.8311 & 0.0021 & 0.0019 & 0.0018 & 0.0020 & 0.0019 & 0.0018 & 0.0017 \\
\hline
\end{tabular}

damper placed either at the first (bottom) or the fifth (top) floor, respectively. The energy dissipation of the damper is varied by considering the three air gaps $G=2 \mathrm{~mm}, 4 \mathrm{~mm}$ and $6 \mathrm{~mm}$. The increase in damping due to the presence of the external damper is clearly demonstrated by the experiments. Furthermore, it is seen that the damping is larger when the damper is located at the top floor and that it increases as the air gap $G$ is reduced.

\subsection{Identification without damper}

Figure 9 shows the estimated damping and stiffness matrix obtained when including the real-valued part of the mode shapes in the identification. The proportional damping matrix previously assumed in the numerical examples in (20) is obtained when only including the real-valued part of the mode shapes in the identification from measurements, see Figure 9 (a). In the identification procedure it has been assumed that the mass matrix is $\mathbf{M}=m \mathbf{I}$, as also considered in (18). The lumped mass $m=m_{f}+m_{c}=2.2 \mathrm{~kg}$ is the sum of the floor mass $m_{f}$ and the effective mass of the columns $m_{c}$. The stiffness matrix in Figure 9 (b) is estimated by the expression in (17), which yields the stiffness matrix in (18) with great numerical accuracy. Figure 10 (a) shows the identified damping matrix for the building model without a damper. However, now the damping matrix is obtained when including the complex mode shapes in the identification. It is found that the structural damping is not proportional. The largest damping component of the matrix in Figure 10 (a) occurs at the first degree-of-freedom. This is partly expected because of the influence from the connection and the interaction between the shear building and shaking table. The changes in the identified stiffness matrix are negligible when the complex mode shapes are included in the identification.

The real parts of the complex mode shapes are illustrated in Figure 11 (a)-(e). In the illustrations, the mode shapes are normalized so that the maximum amplitude of each mode is equal to unity, whereby the imaginary part vanishes at the maximum position. This particular normalization corresponds to a rotation in the complex plane that maximizes the real-valued part of the mode shape [40]. The corresponding imaginary parts of the mode shapes are shown in Figure 11(f)-(j) for the case with no external damper (solid red 

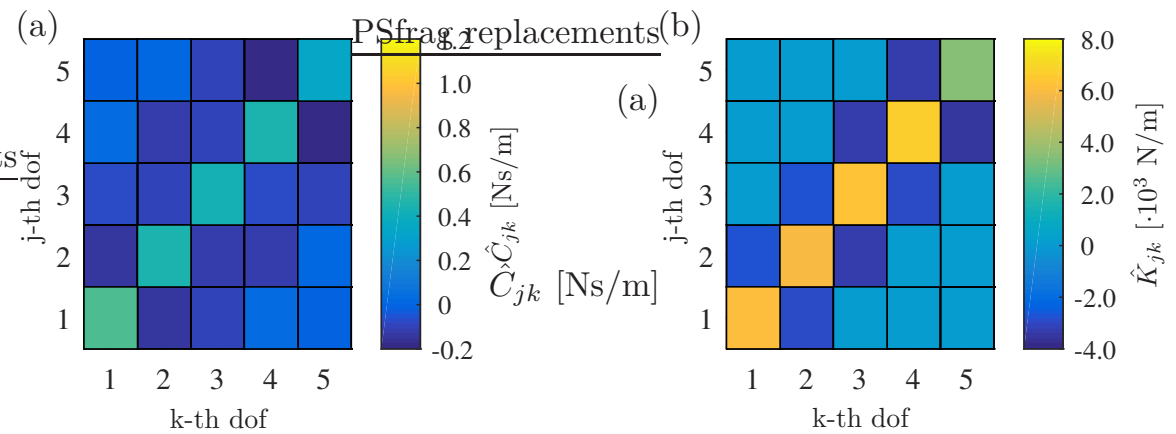

Figure 9: Estimates of the damping and stiffness matrices from real valued mode shapes without damper. (a) Components $\hat{C}_{j k}$ of the damping matrix $\hat{\mathbf{C}}$. (b) Components $\hat{K}_{j k}$ of the stiffness matrix $\hat{\mathbf{K}}$.

curve). They are comparably small due to the limited structural damping in the building model.

The damping matrix is also estimated by Adhikari's method, which again acts as benchmark method. In the experimental analysis the difference in Figure 10 (b) is determined by the error matrix (23), but now with the results from the present direct method relative to the results obtained by the method of Adhikari. The error shows that the damping estimated by the present method is $6 \%$ larger than for Adhikari's method, at the location where the damping magnitude is largest. This tendency is consistent with the more detailed numerical comparison of the two methods in Section 3. Therefore, the damping will in the remainder of this paper only be obtained by the proposed damping identification method in Table 1.

\subsection{Identification of light damping}

The experimental test performed with the eddy current damper placed at the first and the fifth floor yield the damping matrices shown in Figure 12. The first three matrices in (a)-(c) represent the damper at the first floor with air gaps $G=2 \mathrm{~mm}, 4 \mathrm{~mm}$ and $6 \mathrm{~mm}$, respectively. The next three matrices in (d)-(f) are for the damper placed at the top floor. Therefore, the six matrices in the figure correspond to the values presented in Table 2. Initially, it is found that all matrices are nearly symmetric. Furthermore, the particular
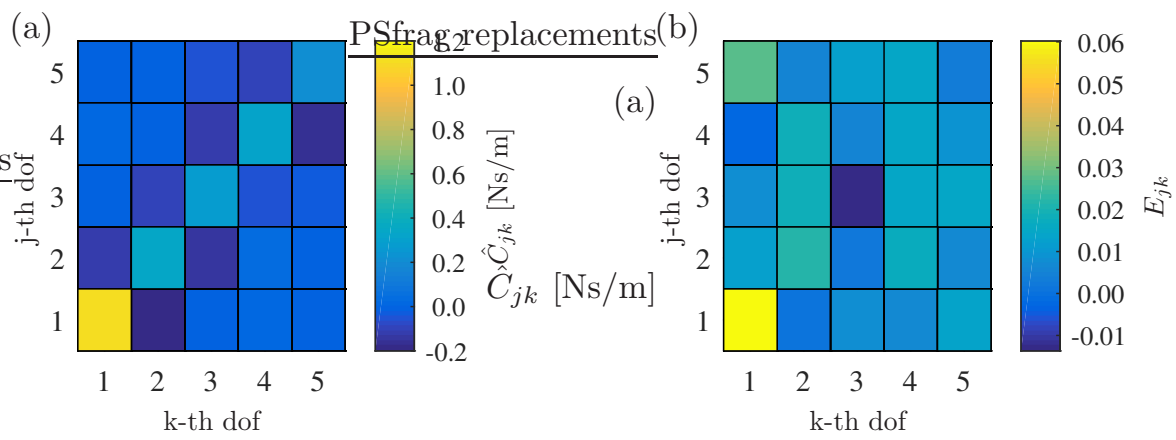

Figure 10: Estimates of the damping from complex valued mode shapes without damper. (a) Components $\hat{C}_{j k}$ of the damping matrix $\hat{\mathbf{C}}$. (b) Components $E_{j k}$ of the relative error matrix obtained by comparing the damping matrix identified with the direct method and Adhikari's method. 


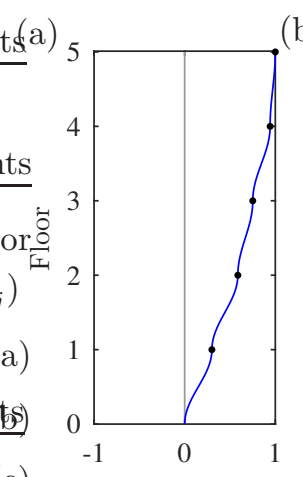

(b)

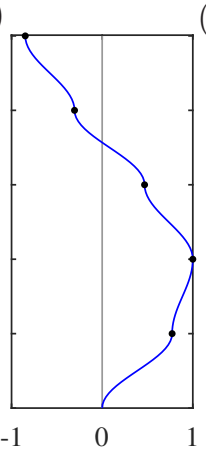

c)

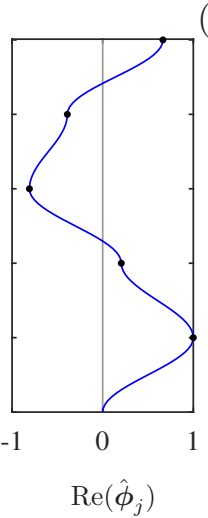

(d)

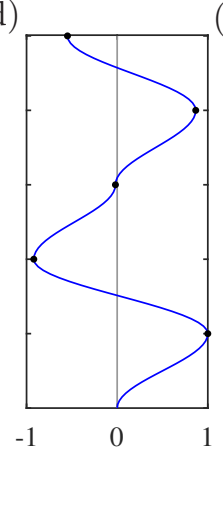

(e)

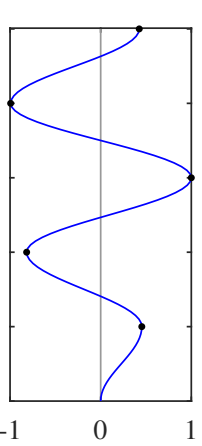

(i)

(g)
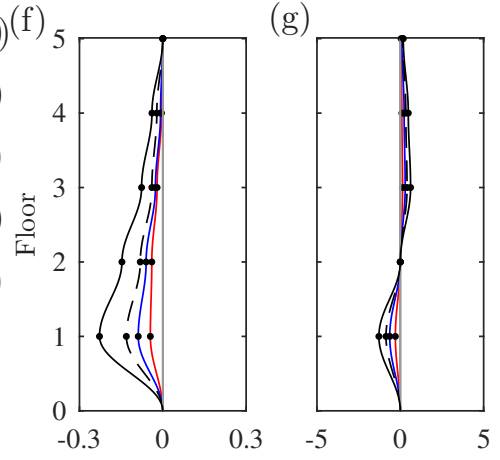

(h)

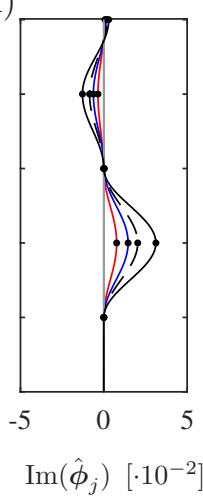

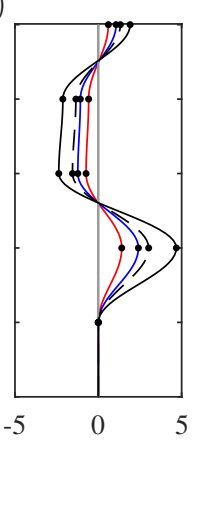

(j)

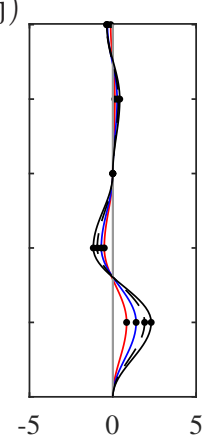

$(\mathrm{k})_{5}$

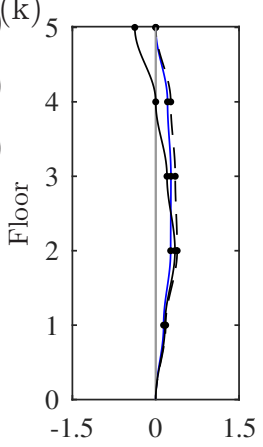

(1)

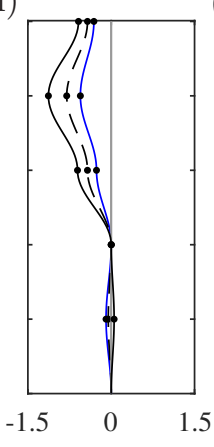

(m)

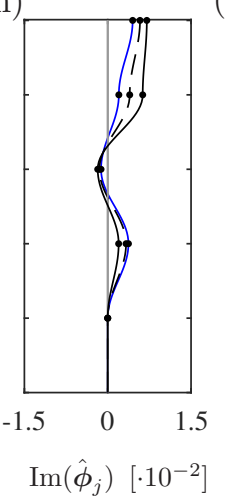

(n)

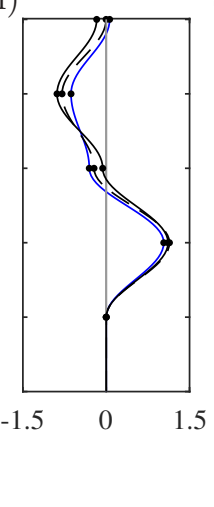

(o)

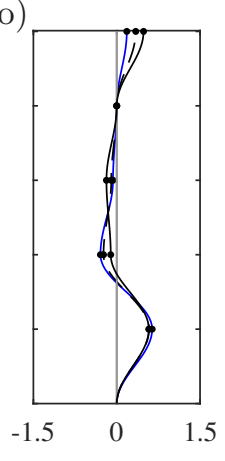

Figure 11: Identified complex-valued mode shapes (•) of the first firve modes. (a)-(e) The real valued part. (f) - (j) The imaginary part with a damper at the first floor and $(\mathrm{k})-(\mathrm{o})$ damper at the fifth floor. Coloring represents an air gap between magnet and floor of $2 \mathrm{~mm}(-), 4 \mathrm{~mm}(--), 6 \mathrm{~mm}(-)$ and damper removed $(-)$.

location of the damper at, either the bottom, or the top floor is clearly identified by the procedure and the magnitude of the damping value decreases consistently with the increase in damper air gap $G$. When the damper is located at the top floor, given in Figures 12 (d) -(f), the structural damping source from Figure 10 (a) is again visible. The real part of the damped vibration modes are basically identical to the mode shapes without damper shown in Figure $11(\mathrm{a})-(\mathrm{e})$. 


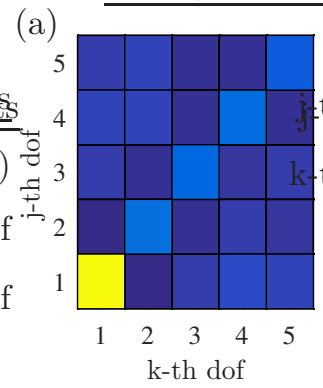

(b)

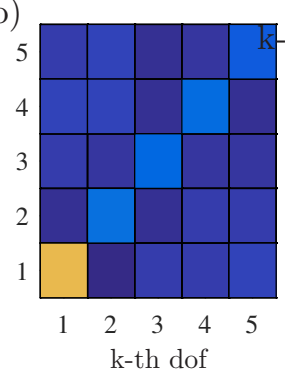

(d)

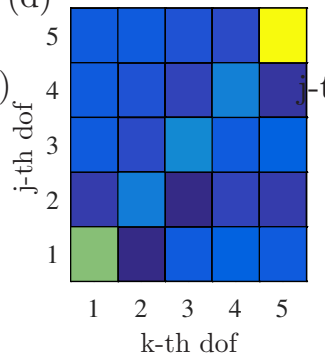

(e)

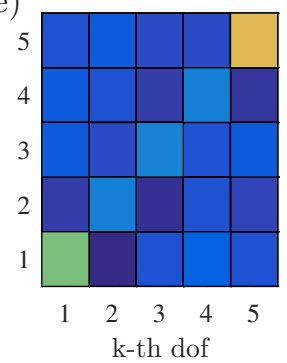

(c)

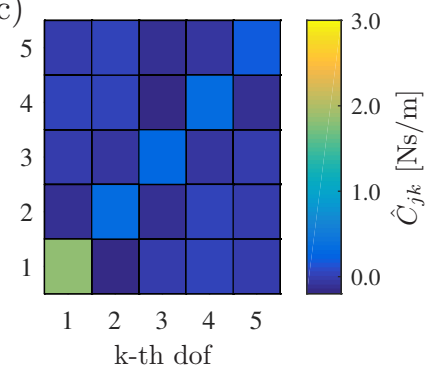

(f)

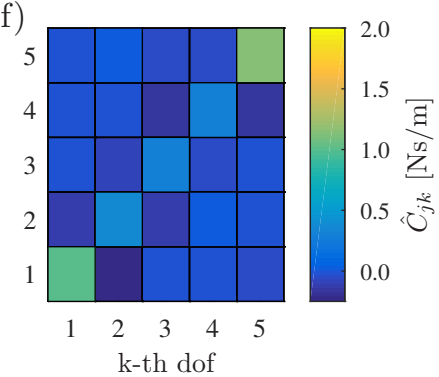

Figure 12: Components $\hat{C}_{j k}$ of the estimated damping matrix $\hat{\mathbf{C}}$ with the damper placed at the first floor with an air gap size of (a) $2 \mathrm{~mm}$, (b) $4 \mathrm{~mm}$ and (c) $6 \mathrm{~mm}$, and the damper placed at the fifth floor with an air gap size of (d) $2 \mathrm{~mm}$, (e) $4 \mathrm{~mm}$ and (f) $6 \mathrm{~mm}$.

The influence of the local damper appears in the imaginary part of the mode shapes, shown in Figure $11(\mathrm{f})-(\mathrm{j})$ for the damper placed at the first floor and in Figure $11(\mathrm{k})-(\mathrm{o})$ for the damper at the fifth floor. For each vibration mode the figure shows the imaginary part with a damper air gap of $G=2 \mathrm{~mm}$ (black solid), $4 \mathrm{~mm}$ (black dashed) and $6 \mathrm{~mm}$ (blue solid). It is seen from the identified complex mode that the imaginary part decreases as the damper air gap increases, and that the amplitude of the imaginary part of the modes is furthermore sensitive to the magnitude of the damper force. For all the imaginary parts of the vibration modes in Figure 11 it is seen that the amplitude of the imaginary part scales with the magnitude of the damper force, whereas the shape is virtually unchanged.

Measurements have also been performed with dampers attached at the second, third and fourth floor. The estimated imaginary parts of the mode shapes and damping matrices are shown in Appendix A. For these cases the location of the damper has also been accurately identified and the magnitude of the damping varies consistently with the air gap. However, the damping matrices with dampers placed at the third and the fourth floor with a small air gap are not symmetric, see Figure A.16 (d) and (g). This inconsistency occurs because of the small vibration amplitudes associated with the nodal point at the third floor in mode four, see Figure 11(d). A non-symmetric damping matrix has similarly been identified when large levels of damping are introduced, as demonstrated in Appendix B, which is also expected to be related to the small vibration amplitudes. Large damping in Appendix B has been obtained by connecting six magnets of the same type, 
as in the present experiment with a single magnet in this section.

\section{Robustness of the identification procedure}

In experimental testing it is important that the test procedure, the data processing and the identification results are robust. Repeatable and converged identification results of the damping matrix and complex mode shapes are, thus, of vital importance. Therefore, experimental tests are preformed to carefully analyze the correlation of repeated dynamic testing and the subsequent identification results. A measure which effectively quantifies the correlation of two sets of mode shape vectors $\phi$ and $\phi^{\prime}$ is the modal assurance criterion (MAC) [26]. The MAC-matrix is defined as

$$
\boldsymbol{\Gamma}=\frac{\left|\sum_{j=1}^{N} \phi_{j}^{\prime} \phi_{j}^{H}\right|^{2}}{\left(\sum_{j=1}^{N} \phi_{j} \phi_{j}^{H}\right)\left(\sum_{j=1}^{N} \phi_{j}^{\prime}{\phi_{j}^{\prime H}}^{H}\right)},
$$

which recovers a unit diagonal matrix when the two sets vectors are fully correlated. The MAC-matrix is denoted as $\boldsymbol{\Gamma}$ and used to separately quantify the correlation of experimental results of the real part and the imaginary part of the complex modes. Numerous measures of correlation and complexity have been established for complex-modes, such as the extended modal assurance criterion and the modal phase collinearity criterion [40]. However, the MAC-matrix defined in (26) is a suitable measure when the real and imaginary parts of the complex mode shapes must be analyzed independently.

\subsection{Repeatability of the identification results}

The dynamic tests of the shear building model with the damper either at the first floor or the fifth floor have been repeated four times. The air gap size is $G=2 \mathrm{~mm}$ in all experiments and the measurement duration of each of the four repeated tests is 3 hours, equivalent to 17928 periods of the first mode. The duration of 3 hours is justified in Subsection 6.2, which discusses the convergence of the imaginary part of the mode shapes with respect to the measurement duration.

The degree to which the experimental results are repeatable is quantified by the correlation between the identified results from each experimental test, obtained by the MAC-matrix through the expression in (26). Firstly, the real parts of the mode shapes identified from each repeated measurement are correlated with each other. The first test is regarded as the reference, and the remaining three tests are then correlated to this reference test. Hereby, three correlation matrices are obtained. The correlations between the realvalued mode shapes from each test yield diagonal MAC-matrices with almost exact unit values, and they are therefore not illustrated. Thus, the identified real-valued part of the mode shapes are linearly independent and reliably identified.

Figure 13 now shows the MAC-matrix for the correlation between the imaginary part of the mode shapes obtained from the four repeated tests, where (a)-(c) are the correlations with the damper placed at the first 
floor and (d)-(f) are with the damper placed at the fifth floor. The dominant values of the MAC matrices appear in the diagonal and they are approximately equal to unity. These MAC matrices are not diagonal, since the imaginary parts in lightly damped systems can be approximated by a linear combination of the real-valued mode shapes, see the expression in (5). The largest off-diagonal terms in the MAC matrices in Figure 13 (a) - (c) occur due to the correlation between the imaginary part of the third and the fourth mode shapes. This is confirmed by the mode shapes illustrated in Figure 11 where (h) and (i) have similar shapes at the second floor. Similarly, the largest off-diagonal terms in the MAC matrices in Figure 13 (d) -(f) when the damper is at the top floor, occur between the imaginary part of the second and the third mode shapes. Again, this is due to the similarity of the mode shape illustrated in Figure 11(l) and (m) at the top floor. The three MAC matrices in Figure $13(\mathrm{a})$-(c) are almost identical, which illustrates a large degree of repeatability in the estimation of the complex mode shapes when the damper is at the first floor. However this is not the case for the MAC matrices in (d)-(f), associated with measurements obtained with the damper at the top floor. The color codes in the figures (d) - (f) indicate a correlation between 0.6 and 1.0 for the fifth mode shape. The repeatability analysis therefore demonstrates that the mode shapes are identified with great assurance, while the magnitude of the imaginary part of the fifth mode shape exhibits a certain degree of variation.

Apart from the mode shapes, the damping matrix has been obtained from each repeated test. The identified value of the damping matrix at the location of the damper is given in Table 3 for all four tests. The largest

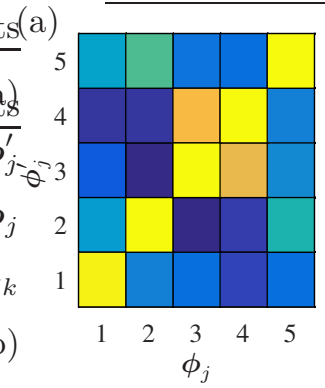

(d)

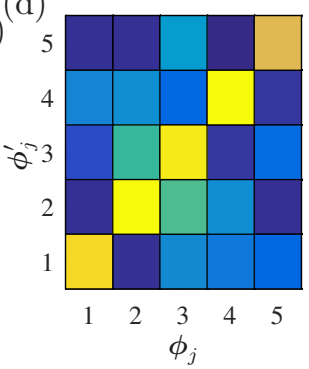

(b)

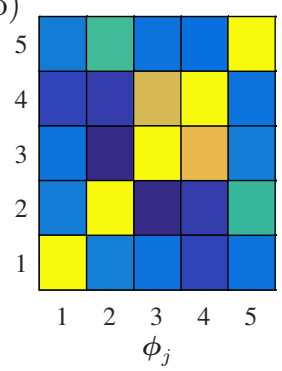

(e)

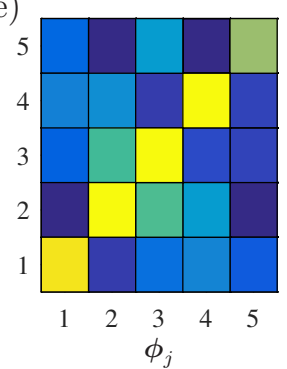

(c)

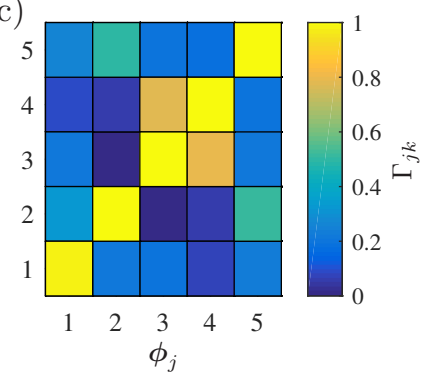

(f)

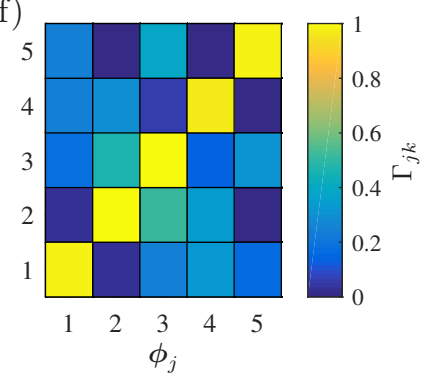

Figure 13: Components $\Gamma_{j k}$ of the MAC-matrix $\boldsymbol{\Gamma}$ between two sets of imaginary values of the complex modes from four repeated tests. (a)-(c) Damper at the first floor with an air gap of $2 \mathrm{~mm}$. (d) -(f) Damper at the fifth floor with an air gap of $2 \mathrm{~mm}$. 
Table 3: Identified components of the damping matrix from repeated test with damper at two locations with an air gap of $2 \mathrm{~mm}$, where the mean value is $\mu$ and standard deviation is $\sigma$.

\begin{tabular}{cccccccc}
\hline Location & & Test 1 & Test 2 & Test 3 & Test 4 & $\mu$ & $\sigma$ \\
\hline First floor & $\hat{C}_{11}[\mathrm{Ns} / \mathrm{m}]$ & 3.28 & 3.25 & 3.26 & 3.33 & 3.28 & 0.04 \\
Fifth floor & $\hat{C}_{55}[\mathrm{Ns} / \mathrm{m}]$ & 1.91 & 1.91 & 2.02 & 2.03 & 1.97 & 0.07
\end{tabular}
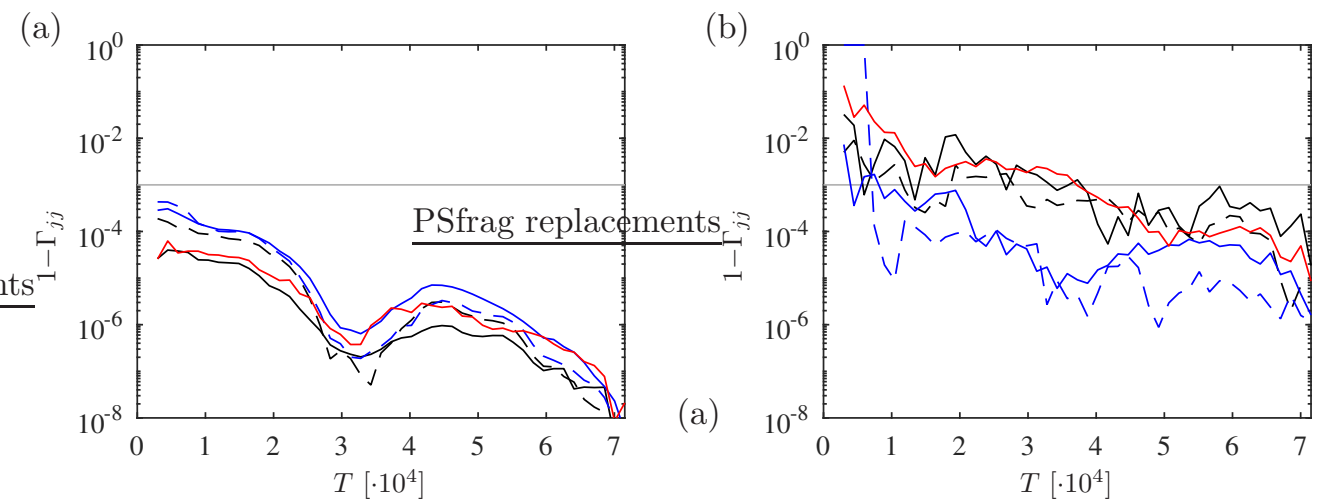

Figure 14: The diagonal components $\Gamma_{j j}$ of the MAC-matrix $\boldsymbol{\Gamma}$ obtained from measurements of duration $T$. Convergence of the (a) real valued part and (b) imaginary part of the estimated complex mode shapes. Coloring $j=1(-), j=2(--), j=3$ $(-), j=4(--), j=5(-)$ and a threshold value of $\Gamma_{j j}=0.99(-)$.

variation relative to the determined mean value is $3.05 \%$, obtained from Test 4 for the damper located at the fifth floor. This is in most cases considered a small uncertainty that is well within a tolerable range for the identification of structural damping.

\subsection{Convergence of the identification results}

The convergence of the estimated complex mode shapes and the damping matrix is analyzed with respect to the duration of the vibration measurements. The measurement duration plays an important role in the estimation of the correlation function matrix, which is the initial input in the identification procedure in Table 1. A finite record length introduces bias and variance errors in the correlation function estimates [28], which then vanishes asymptotically for increasing measurement duration.

The convergence of the identified real and imaginary values of the mode shapes is evaluated by the degree of correlation between the estimates for varying lengths of the measurement duration, with the damper placed at the first floor with an air gap of $G=2 \mathrm{~mm}$. The correlation is obtained by the MAC-matrix given in (26) where the real and imaginary part of the mode shapes are correlated separately, for estimates obtained by an increasing measurement duration. In this case the reference identification results, kept constant in the correlation study and denoted $\phi_{j}^{\prime}$, are obtained from a full 12 hour measurement duration, corresponding to 71500 number of equivalent periods of the first mode. The 12 hour measurement is the longest measurement 


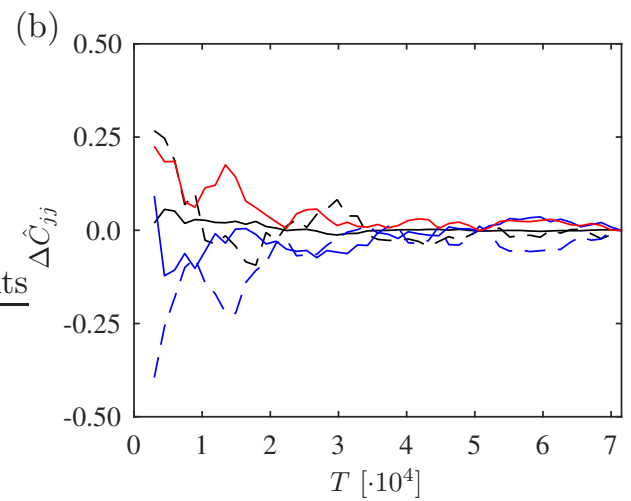

Figure 15: Relative difference of the diagonal components in the estimated damping matrix $\hat{\mathbf{C}}$. Coloring $j=1(-), j=2(--)$, $j=3(-), j=4(--)$ and $j=5(-)$.

duration considered, and thus it is regarded as the measurement which yields converged identification results as the bias and variance error on the correlation function is in this case smallest, compared to identification results from shorter measurement duration. Thus the estimates in $\phi_{j}^{\prime}$ are correlated with the estimates $\phi_{j}$, where the latter are obtained from gradually shorter measurement duration.

The diagonal elements of the MAC-matrix of the convergence study are shown in Figure 14(a) and (b) for the real and imaginary part of the identified mode shapes. In Figure 14 the measurement duration $t$ is normalized by the natural frequency $f_{1}$ of the fundamental mode, whereby $T=t f_{1}$ represents the equivalent number of periods by the fundamental mode with $f_{1}=1.66 \mathrm{~Hz}$. The identified mode shapes are regarded as converged when the correlation reaches a threshold MAC-value of $\Gamma_{j j}=0.99$, as indicated by the horizontal grey line in Figure 14. The correlation of both the identified (a) real and (b) imaginary parts is seen to increase with the measurement duration.

The recommended measurement duration obtained by (25) is introduced as a rule of thumb to obtain a bias error of less than $4 \%$ and a variance error of less than $6 \%$. The recommended duration depends on the product $f_{n} \zeta_{n}$, where $f_{n}$ is the fundamental natural frequency (in Hertz). For the measurements in this convergence study the products $f_{n} \zeta_{n}$ for all modes $n=1$ to 5 are $0.0046 \mathrm{~Hz}, 0.0276 \mathrm{~Hz}, 0.0529 \mathrm{~Hz}, 0.0536 \mathrm{~Hz}$ and $0.0243 \mathrm{~Hz}$, respectively. This implies that the longest measurement duration would be expected for modes 1, 2 and 5. This is consistent with the convergence of the imaginary part shown in Figure 14(b), which also shows that a longer measurement duration is necessary for the imaginary part of modes 1,2 and 5 as they reach the threshold of $\Gamma_{j j}=0.99$ at a slower rate compared to the other modes.

Due to the variability in the estimated imaginary parts of the mode shapes for shorter measurement duration, a similar variability can be expected in the identified damping matrix. The components in the diagonal of the damping matrix $\hat{C}_{j j}$ obtained at a short measurement duration are compared with the diagonal elements $\hat{C}_{j j}^{\prime}$ identified from the longest measurement duration. Figure 15 shows the relative difference $\Delta \hat{C}_{j j}=\frac{\hat{C}_{j j}-\hat{C}_{j j}^{\prime}}{\hat{C}_{j j}^{\prime}}$ of 
the damping matrix component $\hat{C}_{j j}^{\prime}$ from the longest measurement duration and the component $\hat{C}_{j j}$ of the damping matrix from gradually shorter duration. In Figure 15 a relative difference less than $10 \%$ is found when the measurement duration is greater than 40000 number of periods of the first mode. This is equivalent to the number of periods required for the imaginary part of the mode shapes to reach the MAC threshold of 0.99, and it corresponds to approximately seven hours. Thus, a consistent convergence is observed for both the real and imaginary part of the identified complex modes as well as the diagonal values in the damping matrix.

\section{Discussion on damping and complex modes from measurements}

For multiple measurements with light damping some general observations have been made. Firstly, the shape of the imaginary part of the first mode shape is similar to that of the second undamped mode shape, see Figure 11 (f) and (k), Figure A.17(a), (f) and (k) and Figure B.18(a). This has also been discussed in $[15,20]$ and can be related to the product $\frac{\omega_{1} c_{12}^{\prime}}{\omega_{1}^{2}-\omega_{2}^{2}} \mathbf{u}_{2}$ in the sum of (4) being largest for $\phi_{1}$. Secondly, the imaginary part of the first mode shape tends to reach the largest amplitude at the location where the damper is placed. Thus, the first complex mode shape may initially indicate the location of the damping source with good accuracy. However, there are drawbacks of using the scaled imaginary part as an indicator of the spatial location of a damping source. This disadvantage is for cases of the damper location coinciding with the location of the maximum value of the real valued part of the mode shape, as for example illustrated by the imaginary part of the complex mode shape in Figure $11(\mathrm{k})$, for which the maximum value appears at the second floor although the damper acts at the top floor.

Furthermore, a smaller air gap between the magnet and floor plate in the experimental test causes an increase in the modal overlap factor and results in a corresponding increase in the amplitude of the imaginary part of the mode shapes. As an example, the amplitude of the imaginary part of the first mode shape in Figure 11 (f) is 5 times larger at the first floor when the damper is attached with an air gap of $G=2 \mathrm{~mm}$ than without a damper. This observation is agreement with the analytically developed understanding of the sensitivity of the complex mode shapes in [39]. The effect of the increasing modal overlap factor shows similar tendencies for high damping as those mentioned above for light damping, see Figure B.18 in Appendix B.

It is notable from Figure 9 to 13 that the damping and stiffness matrices obtained from experimental data are not strictly symmetric, although the proposed expressions (16) and (17) for the stiffness and damping matrix, respectively, are based on the assumptions of a conservative system. There could be multiple reasons which cause the experimentally identified damping and stiffness matrices to become non-symmetric. If energy dissipation of the experimental building model follows a viscous model then the non-symmetric part of the damping matrix may not be related to the dissipation within the system. The non-symmetric part of the damping matrix could be caused by; 1) the method itself which has been used to obtain the complex modal 
parameters, in this case the ERA, 2) measurement uncertainties such as the coupling observed between the sliding table and the building model, see Figure $8(\mathrm{c}), 3)$ the limitation of reducing the building model to a five degree-of-freedom system although higher modes are present in the measurements, see Figure 8 (c). As a first step to address the practical challenges in identifying a symmetric damping matrix from measurements, it is suggested to increase the mass of the sliding table in the experimental setup. Thereby the effect of the coupling of the table and building model can be minimized. The proposed approach is not applicable for dynamic structures described by a non-viscous damping model, neither does the approach proposed indicate what damping model is most suitable for the measured vibrations of a structure.

\section{Conclusions}

A novel explicit expression for identification of non-classical damping has been derived, which can be applied to recognize the spatial distribution of viscous damping from vibration measurements. The modal parameters and the mass matrix are required in the construction of the damping matrix. The method circumvents the problem of a priori knowledge of the damping in the normalization of the mode shapes, is not limited to lightly damped systems, and can be incorporated in output-only system identification techniques as well as traditional modal testing procedures. Numerical simulations of the random vibration of a five-story shear building model shows that the damping method can identify the location and magnitude of the spatial source of small and large damping.

Dynamic testing of a building model mounted on a shaking table has been carried out, where damping was induced by an eddy current damper. The proposed identification procedure has been applied to measurements of displacements induced by white noise excitation, with dampers at a number of locations and variations in magnitude. The complex-valued mode shapes estimated by the ERA method, turned out to be repeatable with negligible variations between successive measurements. It has been found that the imaginary part of the mode shapes are sensitive to the magnitude of the damper force, where the amplitude increases concurrently with an increase in the magnitude of the damper force. The spatial location of the damping source has been identified with the proposed method from the measurements with both small and large levels of damping. The proposed damping expression does not guarantee symmetry if the identified modal parameters are erroneous or if the damping model is not viscous. Therefore the obtained damping matrix must be used with care in applications. For all results in the present paper, the non-symmetric part of the identified damping matrix is relatively small, whereby it is recommended that the symmetric part is used in a subsequent structural analysis. Although earlier work has projected some skepticism towards the practicality of obtaining complex modes from experimental data [20], the present work demonstrates that complex modes are indeed achievable from measurements and that they can be used to robustly construct the damping matrix for the dynamic structure. 


\section{Acknowledgments}

The financial support by DTU Mechanical Engineering is appreciated, as well as the financial support from Myrhwolds Fond for laboratory equipment. Furthermore, the authors would like to acknowledge the contributions from the department technicians and students in connection with the development of the experimental setup. 


\section{Appendix A. Other damper locations}

The dynamic testing, described in Sections 4 and 5, have also been conducted repeatedly with the damper placed at the second, third and fourth floor of the building mode. The estimated damping matrix from each test with an air gap $G=2 \mathrm{~mm}, 4 \mathrm{~mm}$ and $6 \mathrm{~mm}$, is given in Figure A.16, where (a)-(c) are for the damper placed at the second floor, (d) - (f) are for the damper placed at the third floor and (g) - (i) are for the damper placed at the fourth floor. The color codes in the figures indicate an increase in the damping when the air gap $G$ is reduced. The modal parameters obtained for each test are given in Figure A.17 and Table A.4.

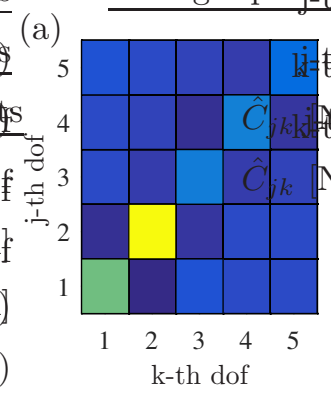

(b)
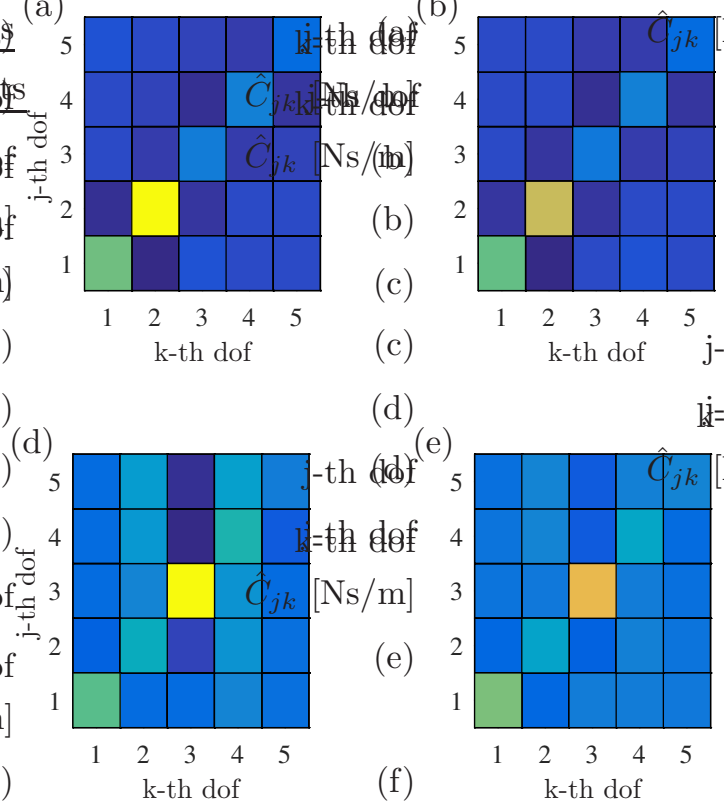

(g)

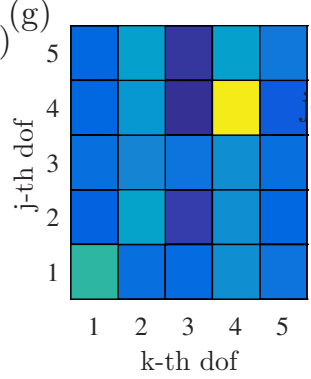

(e)

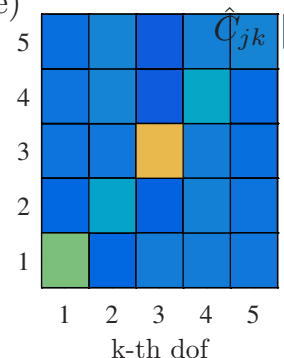

(h)

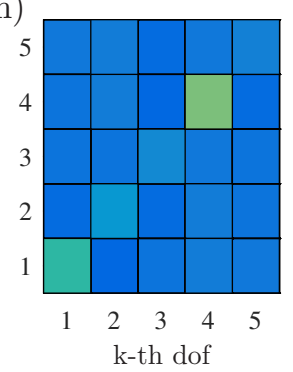

(c)

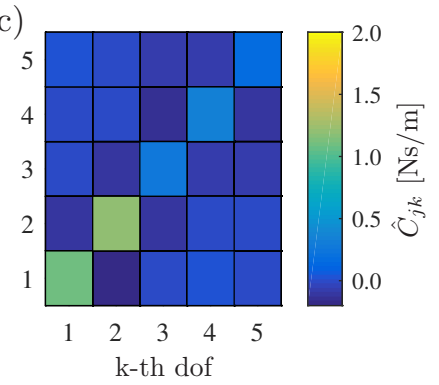

(f)

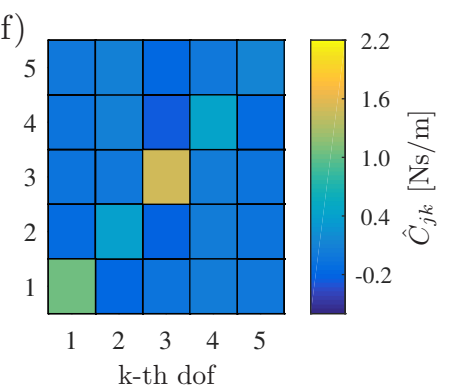

(i)

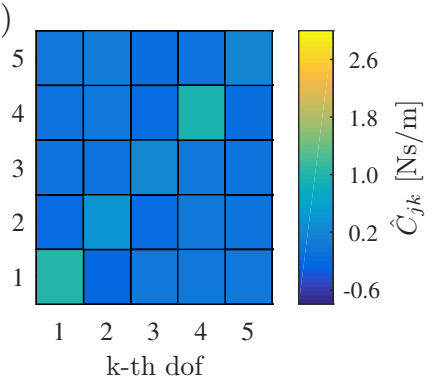

Figure A.16: Components $\hat{C}_{j k}$ of the estimated damping matrix $\hat{\mathbf{C}}$ with the damper placed at the second floor with an air gap size of (a) $2 \mathrm{~mm}$, (b) $4 \mathrm{~mm}$ and (c) $6 \mathrm{~mm}$, the damper placed at the third floor with an air gap size of (d) $2 \mathrm{~mm}$, (e) $4 \mathrm{~mm}$ and (f) $6 \mathrm{~mm}$ and damper placed at the fourth floor with an air gap size of (g) $2 \mathrm{~mm}$, (h) $4 \mathrm{~mm}$ and (i) $6 \mathrm{~mm}$. 

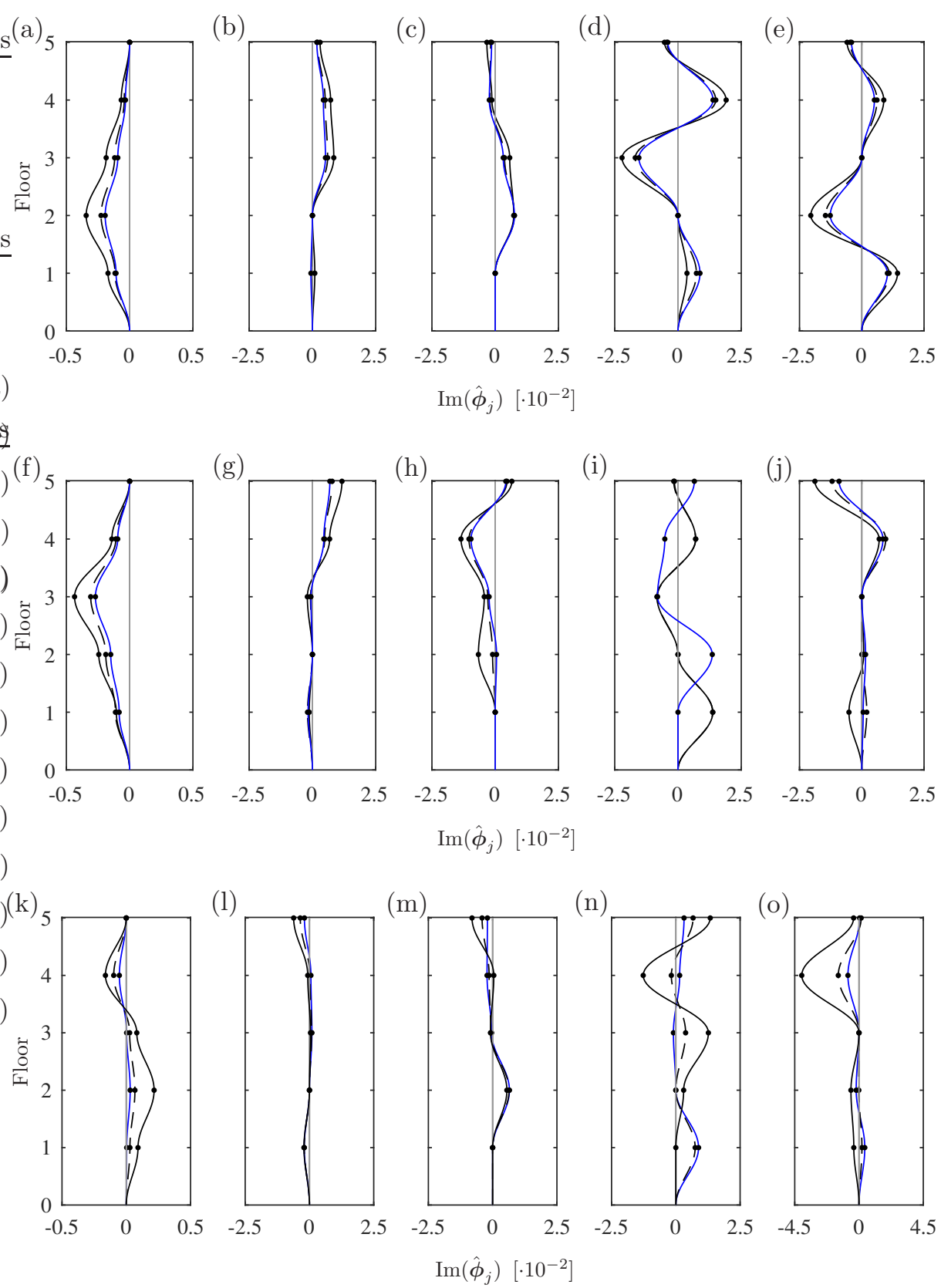

(n)

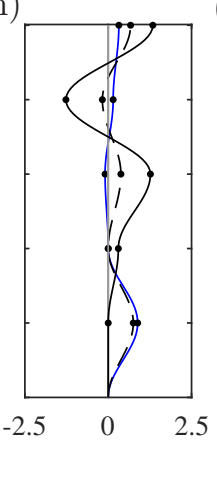

(o)

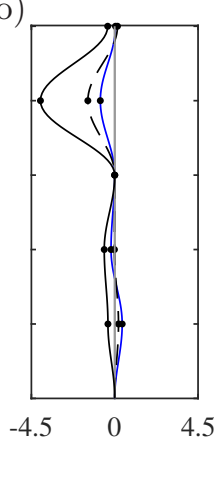

Figure A.17: Identified imaginary part of the first five complex-valued mode shapes $(\bullet)$ from measurements. (a) - (e) Damper at the second floor. (f) $-(\mathrm{j})$ Damper at the third floor. (k)-(o) Damper at the fourth floor. Coloring represents an air gap between magnet and floor of $2 \mathrm{~mm}(-), 4 \mathrm{~mm}(--)$ and $6 \mathrm{~mm}(-)$.

\section{Appendix B. Large external damping}

The dynamic testing described in Section 4 has been repeated with six magnets of the same type, as illustrated in Figure 6. The six magnets compromise an eddy current damper which is placed at the top floor 
Table A.4: Estimated damping ratios $\zeta_{n}$ for the first five modes of the shear building mode from vibration measurements with an eddy current damper at the second, third and fourth floor. The air gap size varies between $2 \mathrm{~mm}$ to $6 \mathrm{~mm}$.

\begin{tabular}{cccccccccc}
\hline$n$ & \multicolumn{3}{c}{ Second floor } & \multicolumn{3}{c}{ Third floor } & \multicolumn{3}{c}{ Fourth floor } \\
& $2 \mathrm{~mm}$ & $4 \mathrm{~mm}$ & $6 \mathrm{~mm}$ & $2 \mathrm{~mm}$ & $4 \mathrm{~mm}$ & $6 \mathrm{~mm}$ & $2 \mathrm{~mm}$ & $4 \mathrm{~mm}$ & $6 \mathrm{~mm}$ \\
\hline 1 & 0.0055 & 0.0040 & 0.0036 & 0.0128 & 0.0088 & 0.0077 & 0.0175 & 0.0092 & 0.0058 \\
2 & 0.0068 & 0.0051 & 0.0046 & 0.0039 & 0.0034 & 0.0032 & 0.0029 & 0.0026 & 0.0024 \\
3 & 0.0029 & 0.0028 & 0.0028 & 0.0059 & 0.0048 & 0.0047 & 0.0035 & 0.0030 & 0.0028 \\
4 & 0.0044 & 0.0037 & 0.0035 & 0.0025 & 0.0025 & 0.0025 & 0.0048 & 0.0037 & 0.0031 \\
5 & 0.0028 & 0.0023 & 0.0022 & 0.0044 & 0.0034 & 0.0031 & 0.0040 & 0.0028 & 0.0023 \\
\hline
\end{tabular}

with an air gap $G=1 \mathrm{~mm}, 2 \mathrm{~mm}$ and $3 \mathrm{~mm}$. Because the top floor has the largest displacements, the largest damper force can be obtained at this location. The identification procedure is identical to that in Section 5. From the identification the modal parameters have been obtained for each test with an air gap between $1 \mathrm{~mm}$ and $3 \mathrm{~mm}$, given in Figure B.18 and Table B.5. For the smallest air gap of $1 \mathrm{~mm}$ between the magnet and the floor plate a damping ratio of $24.65 \%$ is obtained. The estimated damping matrix from each test is given in B.19 (a)-(c), which shows an increase in the damping coefficient when the air gap size decreases.

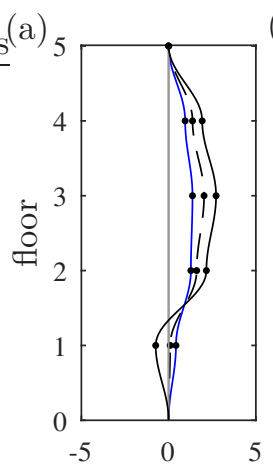

(b)

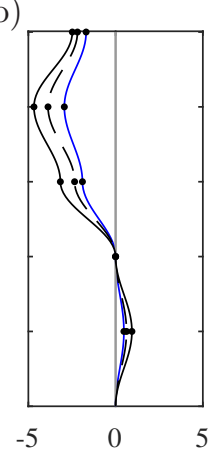

(c)

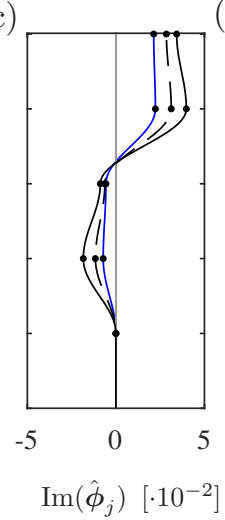

(d)

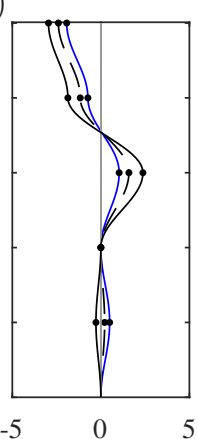

(e)

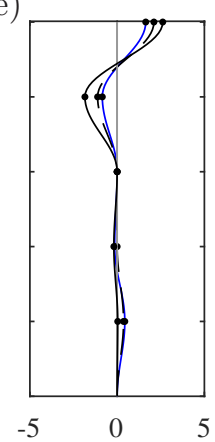

Figure B.18: Estimated imaginary part of the complex-valued mode shape $(\bullet)$ from test with sixs magnets at the fifth floor with an air gap size of $1 \mathrm{~mm}(-), 2 \mathrm{~mm}(--)$ and $3 \mathrm{~mm}(-)$. (a) Mode 1, (b) mode 2, (c) mode 3, (d) mode 4 and (e) mode 5. 
Table B.5: Estimated damping ratios $\zeta_{n}$ for the first five modes of the shear building mode from vibration measurements with six magnets placed at the fifth floor, with an air gap size of $1 \mathrm{~mm}$ to $3 \mathrm{~mm}$.

\begin{tabular}{cccc}
\hline$n$ & $1 \mathrm{~mm}$ & $2 \mathrm{~mm}$ & $3 \mathrm{~mm}$ \\
\hline 1 & 0.2465 & 0.1000 & 0.0546 \\
2 & 0.0176 & 0.0141 & 0.0116 \\
3 & 0.0095 & 0.0080 & 0.0069 \\
4 & 0.0055 & 0.0049 & 0.0044 \\
5 & 0.0030 & 0.0027 & 0.0026 \\
\hline
\end{tabular}
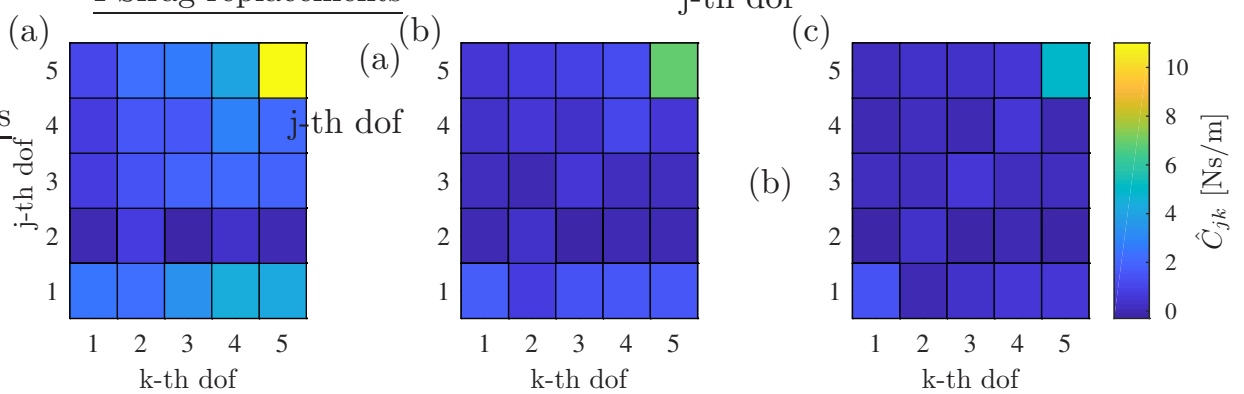

Figure B.19: Components $\hat{C}_{j k}$ of the estimated damping matrix $\hat{\mathbf{C}}$ with the damper placed at the fifth floor with an air gap size of (a) $1 \mathrm{~mm}$, (b) $2 \mathrm{~mm}$ and (c) $3 \mathrm{~mm}$.

\section{References}

[1] E. Reynders, System identification methods for (operational) modal analysis: review and comparison. Archives of Computational Methods in Engineering 2012; 19(1): 51-124.

[2] S.R. Ibrahim, A. Sestieri, Existence and normalization of complex modes in post experimental use in modal analysis. Proceedings of the 13th International Modal Analysis Conference 1995; Vol. 2460.

[3] T. K. Caughey, M. E. J. O'Kelly, Classical normal modes in damped linear dynamic systems. Journal of applied mechanics 1965; 32(3): 583-588.

[4] A. Bhaskar, Mode shapes during asynchronous motion and non-proportionality indices. Journal of Sound and Vibration 1999; 224(1): 1-16.

[5] S. Adhikari, J. Woodhouse, Identification of damping: part 1, viscous damping. Journal of Sound and vibration 2001; 243(1): 43-61.

[6] P. Lancaster, Expression of damping matrices in linear vibration problems. Journal of Aerospace Science 1961; 28: 256. 
[7] S. R. Ibrahim, Dynamic modelling of structures from measured complex modes. AIAA Journal 1983; 21(6): 898901.

[8] C. Minas, D. J. Inman, Identification of a non-proportional damping matrix from incomplete modal information. Journal of Vibration and Acoustics ASME 1991; 113(2): 219224.

[9] D. F. Pilkey, D. J. Inman, An iterative approach to viscous damping matrix identification. In proceedings of the 15th International Modal Analysis Conference IMAC XV 1997; 11521157.

[10] F. Ablitzer, C. Pézerat, J. M. Génevaux, J. Bégué, Identification of stiffness and damping properties of plates by using the local equation of motion. Journal of Sound and Vibration 2014; 333(9): 2454-2468.

[11] C. P. Fritzen, Identication of mass, and stiffness matrices of mechanical systems. Journal of Vibration and Acoustics, ASME 1986; 108: 916.

[12] S. Y. Chen, M. S. Ju, Y. G. Tsuei, Estimation of mass, stiffness and damping matrices form frequency response functions. Journal of Vibration and Acoustics, ASME 1996; 118: 7882.

[13] J. H. Lee, J. Kim, Identification of damping matrices form measured frequency response functions. Journal of Sound and Vibration 2001; 240(3): 545565.

[14] S. Adhikari, Lancasters method of damping identification revisited. Journal of vibration and acoustics $2002 ; 124(4), 617-627$.

[15] A. S. Phani, J. Woodhouse, Viscous damping identification in linear vibration. Journal of Sound and Vibration 2007; 303(3): 475-500.

[16] M. Prandina, J. E. Mottershead, E. Bonisoli, An assessment of damping identification methods. Journal of Sound and Vibration 2009; 323(3): 662-676.

[17] S. Mondal, S. Chakraborty, Identification of non-proportional viscous damping matrix of beams by finite element model updating. Journal of Vibration and Control 2016.

[18] M. Brumat, J. Slavic, M. Boltezar, Spatial damping identification in the frequency domain A theoretical and experimental comparison. Journal of Sound and Vibration 2016; 376: 182-193.

[19] S. Erlicher, P. Argoul, Modal identification of linear non-proportionally damped systems by wavelet transform. Mechanical Systems and Signal Processing 2007; 21(3): 1386-1421.

[20] A. S. Phani, J. Woodhouse, Experimental identification of viscous damping in linear vibration. Journal of Sound and Vibration 2009; 319(3): 832-849. 
[21] J. Juang, R. S. Pappa, An eigensystem realization algorithm for modal parameter identification and model reduction. Journal of guidance, control, and dynamics 1985; 8(5): 620-627.

[22] A. Bajrić, J. Høgsberg, Experimental data for identification of damping and complex modes in structural vibrations. Mendeley Data, 2017.

[23] F. Tisseur, K. Meerbergen, The quadratic eigenvalue problem. Society for Industrial and Applied Mathematics, SIAM review 2001; 43(2): 235-286.

[24] Lord Rayleigh, The theory of sound (Vol. 2). New York: Dover Publications 1945, second edition.

[25] P. Lancaster, U. Prells, Inverse problems for damped vibrating systems. Journal of Sound and Vibration 2005; 283(3): 891-914.

[26] D. J. Ewins, Modal Testing: Theory and Practice, Research Studies Press, Taunton, 1984.

[27] S. Adhikari, J. Woodhouse, Identification of damping part 3: Symmetry preserving methods. Journal of Sound and Vibration 2001, 251(3), 477-490.

[28] A. Bajrić, J. Høgsberg J, F. Rüdinger, Evaluation of damping estimates by automated Operational Modal Analysis for offshore wind turbine tower vibrations. Renewable Energy 2017.

[29] G. H. James, T. G. Carne, J. P. Lauffer, The natural excitation technique (NExT) for modal parameter extraction from operating structures. International Journal of Analytical and Experimental Modal Analysis 1995; 10(4): 260-277.

[30] S. R. Ibrahim, E. C. Mikulcik, A method for direct identification of vibration parameters from the free response The Shock and Vibration Bulletin 1977; 47(4): 183198.

[31] B. Peeters, G. De Roeck, Reference-based stochastic subspace identification for output-only modal analysis. Mechanical Systems and Signal Processing 1999; 13(6): 855-878.

[32] J. S. Lew, J. N. Juang, R. W. Longman, Comparison of several system identification methods for flexible structures. Journal of Sound and Vibration 1993; 167(3): 461-480.

[33] B. A. Pridham, J. C. Wilson, A study of damping errors in correlation-driven stochastic realizations using short data sets. Probabilistic engineering mechanics 2003; 18(1): 61-77.

[34] D. F. Giraldo, W. Song, S. J. Dyke, J. M. Caicedo. Modal identification through ambient vibration: comparative study. Journal of Engineering Mechanics 2009; 135(8): 759-770.

[35] M. Gürgöze, Proportionally damped systems subject to damping modification by several viscous dampers, Journal of Sound and Vibration 2002; 255(2): 407412. 
[36] K. Nagaya, H. Kojima, Y. Karube, H. Kibayashi, Braking forces and damping coefficients of eddy current brakes consisting of cylindrical magnets and plate conductors of arbitrary shape. IEEE Transactions on Magnetics 1984; 20(6): 2136-2145.

[37] G. E. Box, M. E. Muller, A note on the generation of random normal deviates. The annals of mathematical statistics 1958; 29(2): 610-611.

[38] ANSI/ASA S2.47. Vibration of Buildings-Guidelines for the Measurement of Vibrations and Evaluation of Their Effects on Buildings. American National Standard September 10, 1990.

[39] J. Woodhouse, Linear damping models for structural vibration. Journal of Sound and Vibration 1998; 215(3): 547-569.

[40] P. Vacher, B. Jacquier, A. Bucharles, Extensions of the MAC criterion to complex modes. In Proceedings of the international conference on noise and vibration engineering, ISMA 2010, 2713-2726. 